\title{
Global Existence for Stochastic Strongly Dissipative Zakharov Equations
}

\author{
Xueqin Wang $\mathbb{D}^{1},{ }^{1}$ Yadong Shang $\mathbb{D}{ }^{2}$ and Chunlin Lei ${ }^{1}$ \\ ${ }^{1}$ College of Mathematics and Informatics, South China, Agricultural University, Guangzhou, 510642 Guangdong, China \\ ${ }^{2}$ School of Mathematics and Information Science, Guangzhou University, Guangzhou, 510006 Guangdong, China
}

Correspondence should be addressed to Yadong Shang; gzydshang@126.com

Received 17 May 2019; Accepted 22 July 2019; Published 13 February 2020

Academic Editor: Emmanuel Lorin

Copyright (C) 2020 Xueqin Wang et al. This is an open access article distributed under the Creative Commons Attribution License, which permits unrestricted use, distribution, and reproduction in any medium, provided the original work is properly cited.

The stochastic strongly dissipative Zakharov equations with white noise are studied. On the basis of the time uniform a priori estimates, we prove the existence and uniqueness of solutions in energy spaces $\mathscr{E}_{1}$ and $\mathscr{E}_{2}$, by using the standard Galerkin approximation method of stochastic partial differential equations.

\section{Introduction}

The Zakharov system, derived by Zakharov in 1972 [1], describes the interaction between Langmuir (dispersive) and ion acoustic (approximately nondispersive) waves in an unmagnetized plasma. The usual Zakharov system defined in the space $\mathbb{R}^{d+1}$ is given by

$$
\begin{aligned}
& i E_{t}+\Delta E=n E, \\
& n_{t t}-\Delta n=\Delta|E|^{2},
\end{aligned}
$$

where the wave fields $E(x, t)$ and $n(x, t)$ are complex and real, respectively. It has become commonly accepted that the Zakharov system is a general model to govern interaction of dispersive nondispersive waves.

In the past decades, the Zakharov system has been studied by many authors [2-10]. In [4], the authors established globally in time existence and uniqueness of smooth solution for a generalized Zakharov equation in a two-dimensional case for small initial date and proved global existence of smooth solution in one spatial dimension without any small assumption for initial data. In [7, 8], the uniqueness and existence of the global classical solution of the periodic initial value problem for the system of Zakharov equations and the system of generalized Zakharov equations have been proved.
In order to better qualitative agreement, it is necessary to include damping effects or effects of the loss of energy. In a realistic physical system, dissipation must be included into each equation. In [11], the author studied the following dissipative Zakharov equations:

$$
\begin{aligned}
\frac{1}{\lambda^{2}} n_{t t}-n_{x x}-|E|_{x x}^{2}-\alpha n_{t} & =f, \\
i E_{t}+E_{x x}-n E+i \gamma E & =g,
\end{aligned}
$$

where positive constants $\alpha$ and $\gamma$ are damping coefficients and $f$ and $g$ are external forces. The author obtained the long time behavior of (2) and (3) on a bounded interval with initial conditions and homogeneous Dirichlet boundary conditions. The asymptotic behaviors of the solution for (2) and (3) in 1D-2D have been investigated (see [11-13]).

In [14], the authors considered the following strongly dissipative Zakharov equations:

$$
\begin{aligned}
\frac{1}{\lambda^{2}} n_{t t}-n_{x x}-|E|_{x x}^{2}-\alpha n_{t x x} & =f, \\
i E_{t}+E_{x x}-n E+i \gamma E & =g .
\end{aligned}
$$

They studied the Cauchy problem of (4) and (5) and proved the existence of the maximal attractor. 
In recent years, the importance of taking random effects into account in modeling, analyzing, simulating, and predicting complex phenomena has been widely recognized in geophysical and climate dynamics, materials science, chemistry, biology, and other areas [15-18]. Stochastic partial differential equations are appropriate mathematical models for complex systems under random influences or noise. Usually, the noise can be regarded as a simple approximation of turbulence in fluids.

In [19], stochastic dissipative Zakharov equations have been studied on a regular domain $D$ in the Itô sense. The authors proved the existence and uniqueness of solutions. Then, a global random attractor was constructed. Further, the existence of a stationary measure was proved. In [20], the existence and uniqueness of solutions are obtained. Moreover, the asymptotic behaviors of the solutions for the stochastic dissipative quantum Zakharov equations with white noise were also investigated.

In the present paper, we consider the following random forced strongly dissipative Zakharov equations on a regular domain $D$ in the Itô sense:

$$
\begin{array}{r}
\frac{1}{\lambda^{2}} n_{t t}-n_{x x}-|E|_{x x}^{2}-\alpha n_{t x x}=f+\dot{W}_{1}, \\
i E_{t}+E_{x x}-n E+i \gamma E=g+\dot{W}_{2},
\end{array}
$$

where the parameters $\gamma>0$ and $\alpha>0$, and $W_{1}$ and $W_{2}$ are independent $L^{2}(D)$-valued Wiener processes, which will be detailed in the next section. Here, $\dot{W}$ denotes the general derivative of the Wiener processes $W$ with respect to time.

The rest of this paper is organized as follows. In Section 2, some functional setting and some conditions are given. In Section 3, a series of time uniform a priori estimates are given in different energy spaces. Some of the technique of estimates and Itô's formula are used frequently. In Section 4, we obtain the existence and uniqueness of solutions for the stochastic strongly dissipative Zakharov equations by using the standard Galerkin approximation method in different investigated spaces. Various positive constants are denoted by $C$ throughout this paper. Now, we state the main results of the paper.

\section{Preliminary}

In this section, we give a detailed description of the stochastic strongly dissipative Zakharov equations. Let $D=[0, l]$ with $0<l<\infty$. Consider the following strongly dissipative stochastic Zakharov equations on a regular domain $D$ :

$$
\begin{aligned}
\frac{1}{\lambda^{2}} n_{t t}-n_{x x}-|E|_{x x}^{2}-\alpha n_{t x x} & =f+\dot{W}_{1}, \\
i E_{t}+E_{x x}-n E+i \gamma E & =g+\dot{W}_{2}, \quad t \in R^{+}, x \in D,
\end{aligned}
$$

with initial conditions

$$
\left.E\right|_{t=0}=E_{0}(x),\left.n\right|_{t=0}=n_{0}(x),\left.n_{t}\right|_{t=0}=n_{1}(x), \quad x \in D,
$$

and Dirichlet boundary conditions

$$
E=0, n=0, t \in R^{+}, x \in \partial D \text {; }
$$

here, $f, g, \alpha, \gamma$, and $\lambda$ are given, with $\alpha>0$ and $\gamma>0$.

As in [14], to study the solution of strongly dissipative stochastic Zakharov equations, we set $m=n_{t}+\varepsilon n$, where $\varepsilon>0$ is small enough and will be chosen later. So we have the following equations:

$$
\begin{aligned}
& m=n_{t}+\varepsilon n, \\
& \frac{1}{\lambda^{2}} m_{t}-\frac{\varepsilon}{\lambda^{2}} m-\alpha m_{x x}+\frac{\varepsilon^{2}}{\lambda^{2}} n-(1-\varepsilon \alpha) n_{x x} \\
&-|E|_{x x}^{2}=f+\dot{W}_{1}, \\
& i E_{t}+E_{x x}-n E+i \gamma E \\
&=g+\dot{W}_{2}, \quad t \in R^{+}, x \in D, \\
&\left.E\right|_{t=0}=E_{0}(x),\left.n\right|_{t=0}=n_{0}(x),\left.m\right|_{t=0} \\
&=n_{1}(x)+\varepsilon n_{0}(x)=m_{0}(x), \quad x \in D, \\
& E=0, n=0, m=0, t \in R^{+}, x \in \partial D .
\end{aligned}
$$

Here, we give a complete probability space $(\Omega, \mathscr{F}$, $\left.\left\{\mathscr{F}_{t}\right\}_{t \geq 0}, \mathbb{P}\right)$. The expectation operator with respect to $\mathbb{P}$ is denoted by $\mathbb{E}$. The stochastic terms $W_{1}(t)$ and $W_{2}(t)$ on $\left(\Omega, \mathscr{F},\left\{\mathscr{F}_{t}\right\}_{t \geq 0}, \mathbb{P}\right)$ are defined by

$$
W_{1}(t)=q_{1}(x) \omega_{1}(t), W_{2}(t)=q_{2}(x) \omega_{2}(t)
$$

where $\omega_{1}(t)$ is a standard real-valued Wiener process and $\omega_{2}(t)$ is a standard complex-valued Wiener process independent of $\omega_{1}(t)$. In addition, $q_{1}(x)$ and $q_{2}(x)$ are sufficiently smooth functions.

We will work on the usual functional spaces $L^{2}(D), H^{m}$ $(D)$, and $H_{0}^{1}(D)$. The inner product on $L^{2}(D)$ will be denoted by $(u, v)=\operatorname{Re} \int_{D} u(x) \bar{v}(x) d x, u, v \in L^{2}(D)$ and the norm by $\|u\|_{0}=\|u\|_{L^{2}}=\left(\int_{D}|u|^{2} d x\right)^{1 / 2}$. The general $p$-norm of $L^{p}(D)$ $(p \geq 1)$ is denoted by $\|u\|_{L^{p}}=\left(\int_{D}|u|^{p} d x\right)^{1 / p}$. And the norm on $H^{m}(D)$ is denoted by $\|u\|_{m}=\left(\sum_{0 \leq|\alpha| \leq m}\left\|D^{\alpha} u\right\|_{L^{2}(D)}^{2}\right)^{1 / 2}$. The real and imaginary parts of a complex number $A$ are denoted, respectively, by $\operatorname{Re} A$ and $\operatorname{Im} A$.

Now, we define spaces $\mathscr{E}_{i}, i=0,1,2$, as $\mathscr{E}_{0}=H^{-1}(D) \times L^{2}$ $(D) \times H_{0}^{1}(D), \quad \mathscr{E}_{1}=L^{2}(D) \times H_{0}^{1}(D) \times\left(H^{2}(D) \cap H_{0}^{1}(D)\right)$, and $\mathscr{E}_{2}=H_{0}^{1}(D) \times\left(H^{2}(D) \cap H_{0}^{1}(D)\right) \times\left\{u \in H^{3}(D) \cap H_{0}^{1}(D), \triangle u\right.$ $\left.\in H_{0}^{1}(D)\right\}$. Endow $\mathscr{E}_{i}$ with the usual product norm $\|\cdot\|_{\mathscr{E}_{i}}$. Then, $\mathscr{E}_{2} \subset \mathscr{E}_{1} \subset \mathscr{E}_{0}$ with compact embeddings.

In our approach, we need the following lemmas. Let $(\mathscr{X}$, $\left.\|\cdot\|_{\mathscr{X}}\right) \subset\left(\mathscr{Y},\|\cdot\|_{\mathscr{Y}}\right) \subset\left(\mathscr{Z},\|\cdot\|_{\mathscr{X}}\right)$ be three reflective Banach spaces and $\mathscr{X} \subset \mathcal{Y}$ with compact and dense embedding. Define the Banach space

$$
\mathscr{G}=\left\{v: v \in L^{2}(0, T ; \mathscr{X}), \frac{d v}{d t} \in L^{2}(0, T ; \mathscr{Z})\right\},
$$


with

$$
\|v\|_{\mathscr{G}}^{2}=\int_{0}^{T}\|v(s)\|_{\mathscr{X}}^{2} d s+\int_{0}^{T}\left\|\frac{d v}{d t}(s)\right\|_{\mathscr{E}}^{2} d s, \quad v \in \mathscr{G} .
$$

Then, we have the following lemma about compactness result (see [21]).

Lemma 1. If $K$ is bounded in $\mathscr{G}$, then $K$ is precompact in $L^{2}$ $(0, T ; \mathscr{Y})$.

Another lemma is needed for some maximal estimates on the stochastic integral. Assume $U$ and $H$ are separable Hilbert spaces, $W$ is a $Q$-Wiener process on $U_{0}$ with $U_{0}=Q^{1 / 2} U$. And let $L_{2}^{0}=L_{2}^{0}\left(U_{0}, H\right)$ be the space of Hilbert-Schmidt operators from $U_{0}$ to $H$. We have the following results (see [22]).

Lemma 2. For any $r \geq 1$ and any $L_{2}^{0}$-valued predictable process $\Phi(t), t \in[0, T]$, we have

$$
\begin{aligned}
& \mathbb{E} \int_{0}^{t} \Phi(s) d W(s)=0, \\
& \mathbb{E}\left(\sup _{s \in[0, t]}\left|\int_{0}^{s} \Phi(\sigma) d W(\sigma)\right|^{2 r}\right) \\
& \leq c_{r} \sup _{s \in[0, t]} \mathbb{E}\left(\left|\int_{0}^{s} \Phi(\sigma) d W(\sigma)\right|^{2 r}\right) \\
& \leq C_{r} \mathbb{E}\left(\mid \int_{0}^{t}\|\Phi(s)\|_{L_{2}^{o}}^{2} d s\right)^{r},
\end{aligned}
$$

where $c_{r}$ and $C_{r}$ are some positive constants dependent on $r$.

\section{Time Uniform A Priori Estimates}

In this section, in the sense of expectation, we give a priori estimates for $\left(n_{t}, n, E\right)$ in different spaces $\mathscr{E}_{0}, \mathscr{E}_{1}$, and $\mathscr{E}_{2}$.

Lemma 3. Assume $g, E_{0}, q_{2} \in L^{2}(D)$. Then, for any $T>0$ and $p \geq 1, E \in L^{2 p}\left(\Omega ; L^{\infty}\left(0, T ; L^{2}(D)\right)\right) \cap L^{\infty}\left(0, \infty ; L^{2 p}(\Omega\right.$, $\left.\left.L^{2}(D)\right)\right)$.

Proof. Applying Itô's formula to $\|E\|_{0}^{2}$, by (13), we have

$$
\begin{aligned}
\frac{d}{d t}\|E\|_{0}^{2}= & 2 \operatorname{Re} \int_{D} E_{t} \bar{E} d x+\left\|q_{2}\right\|_{0}^{2} \\
= & -2 \gamma\|E\|_{0}^{2}+2 \operatorname{Im} \int_{D} g \bar{E} d x \\
& +2 \operatorname{Im} \int_{D} \dot{W}_{2} \bar{E} d x+\left\|q_{2}\right\|_{0}^{2} .
\end{aligned}
$$
obtain

$$
\begin{aligned}
\|E\|_{0}^{2}= & \left\|E_{0}\right\|_{0}^{2}-2 \gamma \int_{0}^{t}\|E(s)\|_{0}^{2} d s+2 \int_{0}^{t} \operatorname{Im} \int_{D} g \bar{E} d x d s \\
& +2 \int_{0}^{t} \operatorname{Im} \int_{D} \dot{W}_{2} \bar{E} d x d s+\left\|q_{2}\right\|_{0}^{2} t \\
\leq & \left\|E_{0}\right\|_{0}^{2}-2 \gamma \int_{0}^{t}\|E(s)\|_{0}^{2} d s+2 \int_{0}^{t}\|g\|_{0}\|E\|_{0} d s \\
& +2 \int_{0}^{t} \operatorname{Im} \int_{D} \dot{W}_{2} \bar{E} d x d s+\left\|q_{2}\right\|_{0}^{2} t \\
\leq & \left\|E_{0}\right\|_{0}^{2}-\gamma \int_{0}^{t}\|E(s)\|_{0}^{2} d s+\left(\frac{1}{\gamma}\|g\|_{0}^{2}+\left\|q_{2}\right\|_{0}^{2}\right) t \\
& +2 \int_{0}^{t} \operatorname{Im} \int_{D} \dot{W}_{2} \bar{E} d x d s .
\end{aligned}
$$

Taking expectation on both sides of (21), by Lemma 2 , we get

$$
\mathbb{E}\|E\|_{0}^{2} \leq \mathbb{E}\left\|E_{0}\right\|_{0}^{2}-\gamma \mathbb{E} \int_{0}^{t}\|E(s)\|_{0}^{2} d s+\left(\frac{1}{\gamma}\|g\|_{0}^{2}+\left\|q_{2}\right\|_{0}^{2}\right) t .
$$

Then, by Gronwall inequality, we get

$$
\mathbb{E}\|E\|_{0}^{2} \leq e^{-\gamma t} \mathbb{E}\left\|E_{0}\right\|_{0}^{2}+\frac{1}{\gamma^{2}}\|g\|_{0}^{2}+\frac{1}{\gamma}\left\|q_{2}\right\|_{0}^{2} \leq C,
$$

where $C$ is independent of $T$.

By (23), we obtain $E \in L^{\infty}\left(0, \infty ; L^{2}\left(\Omega, L^{2}(D)\right)\right)$.

Taking the supremum and expectation on both sides of (21), by Lemma 2 and (23), for any $T>0$, there exists a positive constant $C_{T}$; we have

$$
\begin{aligned}
\underset{0 \leq t \leq T}{\mathbb{E} \sup _{0}\|E\|_{0}^{2} \leq} & \mathbb{E}\left\|E_{0}\right\|_{0}^{2}+\mathbb{E} \sup _{0 \leq t \leq T}\left|\int_{0}^{t} \operatorname{Im} \int_{D} \dot{W}_{2} \bar{E} d x d s\right|^{2} \\
& +\left(\frac{1}{\gamma}\|g\|_{0}^{2}+\left\|q_{2}\right\|_{0}^{2}\right) T+1 \\
\leq & \mathbb{E}\left\|E_{0}\right\|_{0}^{2}+C\left\|q_{2}\right\|_{0}^{2} \mathbb{E} \int_{0}^{T}\|E\|_{0}^{2} d s \\
& +\left(\frac{1}{\gamma}\|g\|_{0}^{2}+\left\|q_{2}\right\|_{0}^{2}\right) T+1 \\
\leq & C_{T}\left(\mathbb{E}\left\|E_{0}\right\|_{0}^{4}+\|g\|_{0}^{4}+\left\|q_{2}\right\|_{0}^{4}+1\right) \\
\leq & C_{T}\left(E_{0}, g, q_{2}\right)
\end{aligned}
$$

where $C_{T}\left(E_{0}, g, q_{2}\right)$ is a constant depending on $\left\|E_{0}\right\|_{0},\|g\|_{0}$, $\left\|q_{2}\right\|_{0}$ and $T$.

By (24), we obtain $E \in L^{2}\left(\Omega ; L^{\infty}\left(0, T ; L^{2}(D)\right)\right)$.

By the above estimates, we can further give an estimate of $\|E\|_{0}^{2 p}$ for any $p \geq 1$. Now applying Itô's formula, Young inequality, and Hölder inequality, we have 


$$
\begin{aligned}
& \frac{d}{d t}\|E\|_{0}^{2 p}=p\|E\|_{0}^{2(p-1)} \frac{d}{d t}\|E\|_{0}^{2}+2 p(p-1)\|E\|_{0}^{2(p-1)}\left\|q_{2}\right\|_{0}^{2} \\
& =p\|E\|_{0}^{2(p-1)}\left(2 \operatorname{Re} \int_{D} E_{t} \bar{E} d x+\left\|q_{2}\right\|_{0}^{2}\right) \\
& +2 p(p-1)\|E\|_{0}^{2(p-1)}\left\|q_{2}\right\|_{0}^{2} \\
& =p\|E\|_{0}^{2(p-1)}\left(-2 \gamma\|E(t)\|_{0}^{2}\right) \\
& +2 p\|E\|_{0}^{2(p-1)} \operatorname{Im} \int_{D} g \bar{E} d x \\
& +2 p\|E\|_{0}^{2(p-1)} \operatorname{Im} \int_{D} \dot{W}_{2} \bar{E} d x \\
& +p(2 p-1)\|E\|_{0}^{2(p-1)}\left\|q_{2}\right\|_{0}^{2} \\
& \leq-2 \gamma p\|\mathrm{E}\|_{0}^{2 p}+2 p\|E\|_{0}^{2(p-1)}\|g\|_{0}\|E\|_{0} \\
& +2 p\|E\|_{0}^{2(p-1)} \operatorname{Im} \int_{D} \dot{W}_{2} \bar{E} d x \\
& +p(2 p-1)\|E\|_{0}^{2(p-1)}\left\|q_{2}\right\|_{0}^{2} \\
& \leq-\gamma p\|E\|_{0}^{2 p}+\frac{p}{\gamma}\|E\|_{0}^{2(p-1)}\|g\|_{0}^{2} \\
& +2 p\|E\|_{0}^{2(p-1)} \operatorname{Im} \int_{D} \dot{W}_{2} \bar{E} d x \\
& +p(2 p-1)\|E\|_{0}^{2(p-1)}\left\|q_{2}\right\|_{0}^{2} \\
& \leq-\gamma p\|E\|_{0}^{2 p}+\frac{\gamma p}{2}\|E\|_{0}^{2 p}+2 p\|E\|_{0}^{2(p-1)} \operatorname{Im} \int_{D} \dot{W}_{2} \bar{E} d x \\
& +C\left(\|g\|_{0},\left\|q_{2}\right\|_{0}\right)=-\frac{\gamma p}{2}\|E\|_{0}^{2 p} \\
& +2 p\|E\|_{0}^{2(p-1)} \operatorname{Im} \int_{D} \dot{W}_{2} \bar{E} d x+C\left(\|g\|_{0},\left\|q_{2}\right\|_{0}\right) .
\end{aligned}
$$

Integrating (25) from 0 to $t$, we obtain

$$
\begin{aligned}
\|E\|_{0}^{2 p} \leq & \left\|E_{0}\right\|_{0}^{2 p}-\frac{\gamma p}{2} \int_{0}^{t}\|E\|_{0}^{2 p} d s+C\left(\|g\|_{0},\left\|q_{2}\right\|_{0}\right) t \\
& +2 p \int_{0}^{t}\|E\|_{0}^{2(p-1)} \operatorname{Im} \int_{D} \dot{W}_{2} \bar{E} d x d s .
\end{aligned}
$$

Taking expectation on both sides of (26), we get

$$
\begin{gathered}
\mathbb{E}\|E\|_{0}^{2 p} \leq \mathbb{E}\left\|E_{0}\right\|_{0}^{2 p}-\frac{\gamma p}{2} \mathbb{E} \int_{0}^{t}\|E\|_{0}^{2 p} d s \\
+C\left(\|g\|_{0},\left\|q_{2}\right\|_{0}\right) t .
\end{gathered}
$$

Then, by Gronwall inequality, we get

$$
\mathbb{E}\|E\|_{0}^{2 p} \leq e^{-\gamma p t / 2} \mathbb{E}\left\|E_{0}\right\|_{0}^{2 p}+C\left(\|g\|_{0},\left\|q_{2}\right\|_{0}\right) \leq C\left(g, q_{2}\right),
$$

where $C\left(g, q_{2}\right)$ is independent of $T$.

By (28), we obtain $E \in L^{\infty}\left(0, \infty ; L^{2 p}\left(\Omega, L^{2}(D)\right)\right)$.

Taking the supremum and expectation on both sides of (26), by Lemma 2 and (28), for any $T>0$, there exists a positive constant $C_{T}$; we have

$$
\begin{aligned}
\sup _{0 \leq t \leq T}\|E\|_{0}^{2 p} \leq & \mathbb{E}\left\|E_{0}\right\|_{0}^{2 p}+C\left(\|g\|_{0},\left\|q_{2}\right\|_{0}\right) T+1 \\
& +p^{2} \mathbb{E} \sup _{0 \leq t \leq T}\left|\int_{0}^{t}\|E\|_{0}^{2(p-1)} \operatorname{Im} \int_{D} \dot{W}_{2} \bar{E} d x d s\right|^{2} \\
\leq & \mathbb{E}\left\|E_{0}\right\|_{0}^{2 p}+C\left(\|g\|_{0},\left\|q_{2}\right\|_{0}\right) T+1 \\
& +C p^{2}\left\|q_{2}\right\|_{0}^{2} \mathbb{E} \int_{0}^{T}\|E\|_{0}^{4 p-2} d s \\
\leq & C_{T}\left(\left\|E_{0}\right\|_{0},\|g\|_{0},\left\|q_{2}\right\|_{0}\right) \leq C_{T}\left(E_{0}, g, q_{2}\right)
\end{aligned}
$$

where $C_{T}\left(E_{0}, g, q_{2}\right)$ is a constant depending on $\left\|E_{0}\right\|_{0},\|g\|_{0}$, $\left\|q_{2}\right\|_{0}$ and $T$.

By (29), we obtain $E \in L^{2 p}\left(\Omega ; L^{\infty}\left(0, T ; L^{2}(D)\right)\right)$.

Lemma 3 is proved completely.

Lemma 4. Assume $\left(n_{1}, n_{0}, E_{0}\right) \in \mathscr{E}_{0}, f, q_{1} \in H^{-1}(D)$ and $g$, $q_{2} \in H_{0}^{1}(D)$. Then, for any $T>0$ and $p \geq 1,\left(n_{t}, n, E\right) \in L^{2 p}$ $\left(\Omega ; L^{\infty}\left(0, T ; \mathscr{E}_{0}\right)\right) \cap L^{\infty}\left(0, \infty ; L^{2 p}\left(\Omega, \mathscr{E}_{0}\right)\right)$.

Proof. Applying Itô's formula to $\|m\|_{-1}^{2}$, by (12), we have

$$
\begin{aligned}
\frac{1}{2 \lambda^{2}} \frac{d}{d t}\|m\|_{-1}^{2}= & \int_{D} \frac{1}{\lambda^{2}} m_{t}(-\Delta)^{-1} m d x+\frac{\lambda^{2}}{2}\left\|q_{1}\right\|_{-1}^{2}=\frac{\varepsilon}{\lambda^{2}}\|m\|_{-1}^{2} \\
& -\alpha\|m\|_{0}^{2}-\frac{\varepsilon^{2}}{\lambda^{2}} \int_{D}(-\Delta)^{-1 / 2} n(-\Delta)^{-1 / 2} m d x \\
& -(1-\varepsilon \alpha) \int_{D} n m d x-\int_{D}|E|^{2} m d x \\
& +\int_{D} f(-\Delta)^{-1} m d x+\int_{D} \dot{W}_{1}(-\Delta)^{-1} m d x \\
& +\frac{\lambda^{2}}{2}\left\|q_{1}\right\|_{-1}^{2} .
\end{aligned}
$$

Since $m=n_{t}+\varepsilon n$, from (30), we obtain

$$
\begin{aligned}
\frac{d}{d t}[ & \left.\frac{1}{2 \lambda^{2}}\|m\|_{-1}^{2}+\frac{1}{2}(1-\varepsilon \alpha)\|n\|_{0}^{2}+\frac{\varepsilon^{2}}{2 \lambda^{2}}\|n\|_{-1}^{2}\right] \\
= & \frac{\varepsilon}{\lambda^{2}}\|m\|_{-1}^{2}-\alpha\|m\|_{0}^{2}-\frac{\varepsilon^{3}}{\lambda^{2}}\|n\|_{-1}^{2}-\varepsilon(1-\varepsilon \alpha)\|n\|_{0}^{2} \\
& -\int_{D}|E|^{2} m d x+\int_{D} f(-\Delta)^{-1} m d x \\
& +\int_{D} \dot{W}_{1}(-\Delta)^{-1} m d x+\frac{\lambda^{2}}{2}\left\|q_{1}\right\|_{-1}^{2} \\
\leq & \left(\frac{\varepsilon}{\lambda^{2}}-\alpha \lambda_{1}\right)\|m\|_{-1}^{2}-\frac{\varepsilon^{3}}{\lambda^{2}}\|n\|_{-1}^{2}-\varepsilon(1-\varepsilon \alpha)\|n\|_{0}^{2} \\
& -\int_{D}|E|^{2} m d x+\int_{D} f(-\Delta)^{-1} m d x \\
& +\int_{D} \dot{W}_{1}(-\Delta)^{-1} m d x+\frac{\lambda^{2}}{2}\left\|q_{1}\right\|_{-1}^{2} .
\end{aligned}
$$

where $\lambda_{1}$ is the first eigenvalue of $-\Delta$. 
By (31), we get

$$
\begin{aligned}
& \frac{d}{d t}\left[\frac{1}{2 \lambda^{2}}\|m\|_{-1}^{2}+\frac{1}{2}(1-\varepsilon \alpha)\|n\|_{0}^{2}+\frac{\varepsilon^{2}}{2 \lambda^{2}}\|n\|_{-1}^{2}\right] \\
& \quad+\left(\alpha \lambda_{1}-\frac{\varepsilon}{\lambda^{2}}\right)\|m\|_{-1}^{2}+\varepsilon(1-\varepsilon \alpha)\|n\|_{0}^{2}+\frac{\varepsilon^{3}}{\lambda^{2}}\|n\|_{-1}^{2} \\
& \leq-\int_{D}|E|^{2} m d x+\int_{D} f(-\Delta)^{-1} m d x+\frac{\lambda^{2}}{2}\left\|q_{1}\right\|_{-1}^{2} \\
& \quad+\int_{D} \dot{W}_{1}(-\Delta)^{-1} m d x .
\end{aligned}
$$

Applying Itô's formula to $\left\|E_{x}\right\|_{0}^{2}$, by (13), we have

$$
\frac{d}{d t}\left\|E_{x}\right\|_{0}^{2}=2 \operatorname{Re} \int_{D} E_{x} \bar{E}_{t x} d x+\left\|q_{2}\right\|_{1}^{2}
$$

Substituting $E_{x x}=-i E_{t}+n E-i \gamma E+g+\dot{W}_{2}$ into (33), we have

$$
\begin{aligned}
\frac{d}{d t}\left\|E_{x}\right\|_{0}^{2}= & -2 \operatorname{Re} \int_{D} E_{x x} \bar{E}_{t} d x+\left\|q_{2}\right\|_{1}^{2} \\
= & -2 \operatorname{Re} \int_{D}\left(n E-i \gamma E+g+\dot{W}_{2}\right) \bar{E}_{t} d x+\left\|q_{2}\right\|_{1}^{2} \\
= & -2 \operatorname{Re} \int_{D} n E \bar{E}_{t} d x+2 \operatorname{Re} \int_{D} \gamma \bar{E}\left(-i E_{t}\right) d x \\
& -2 \operatorname{Re} \int_{D} g \bar{E}_{t} d x-2 \operatorname{Re} \int_{D} \dot{W}_{2} \bar{E}_{t} d x+\left\|q_{2}\right\|_{1}^{2} \\
= & -2 \operatorname{Re} \int_{D} n E \bar{E}_{t} d x-2 \gamma\left\|E_{x}\right\|_{0}^{2}-2 \gamma \int_{D} n|E|^{2} d x \\
& -2 \operatorname{Re} \int_{D} \gamma \bar{E}_{g} d x-2 \operatorname{Re} \int_{D} \gamma \bar{E} \dot{W}_{2} d x \\
& -2 \operatorname{Re} \int_{D} g \bar{E}_{t} d x-2 \operatorname{Re} \int_{D} \dot{W}_{2} \bar{E}_{t} d x+\left\|q_{2}\right\|_{1}^{2} \\
= & -2 \operatorname{Re} \int_{D} n E \bar{E}_{t} d x-2 \gamma\left\|E_{x}\right\|_{0}^{2}-2 \gamma \int_{D} n|E|^{2} d x \\
& +\left\|q_{2}\right\|_{1}^{2}-2 \operatorname{Re} \int_{D}\left(\gamma \bar{E}+\bar{E}_{t}\right) g d x \\
& -2 \operatorname{Re} \int_{D}\left(\gamma \bar{E}+\bar{E}_{t}\right) \dot{W}_{2} d x .
\end{aligned}
$$

In addition, applying Itô's formula to $\int_{D} n|E|^{2} d x$, we have

$\frac{d}{d t} \int_{D} n|E|^{2} d x=\int_{D} n_{t}|E|^{2} d x+2 \operatorname{Re} \int_{D} n E \bar{E}_{t} d x+\int_{D} n\left|q_{2}\right|^{2} d x$
Substituting (35) into (34), we obtain

$$
\begin{aligned}
\frac{d}{d t}\left(\left\|E_{x}\right\|_{0}^{2}+\int_{D} n|E|^{2} d x\right) \\
=-2 \gamma\left\|E_{x}\right\|_{0}^{2}-2 \gamma \int_{D} n|E|^{2} d x+\left\|q_{2}\right\|_{1}^{2} \\
\quad-2 \operatorname{Re} \int_{D}\left(\gamma \bar{E}+\bar{E}_{t}\right) g d x-2 \operatorname{Re} \int_{D}\left(\gamma \bar{E}+\bar{E}_{t}\right) \dot{W}_{2} d x \\
\quad+\int_{D} n_{t}|E|^{2} d x+\int_{D} n\left|q_{2}\right|^{2} d x \\
=-2 \gamma\left\|E_{x}\right\|_{0}^{2}-(2 \gamma+\varepsilon) \int_{D} n|E|^{2} d x+\left\|q_{2}\right\|_{1}^{2} \\
\quad+\int_{D} n\left|q_{2}\right|^{2} d x-2 \operatorname{Re} \int_{D}\left(\gamma \bar{E}+\bar{E}_{t}\right) g d x \\
\quad-2 \operatorname{Re} \int_{D}\left(\gamma \bar{E}+\bar{E}_{t}\right) \dot{W}_{2} d x+\int_{D} m|E|^{2} d x .
\end{aligned}
$$

Taking $0<\varepsilon \leq \min \left\{1 / \alpha, \alpha \lambda_{1} \lambda^{2} / 2\right\}$, and by (32) and (36), we have

$$
\begin{aligned}
& \frac{d}{d t}\left[\frac{1}{2 \lambda^{2}}\|m\|_{-1}^{2}+\frac{1}{2}(1-\varepsilon \alpha)\|n\|_{0}^{2}+\frac{\varepsilon^{2}}{2 \lambda^{2}}\|n\|_{-1}^{2}\right] \\
& +\frac{d}{d t}\left(\left\|E_{x}\right\|_{0}^{2}+\int_{D} n|E|^{2} d x\right)+\frac{\varepsilon}{\lambda^{2}}\|m\|_{-1}^{2} \\
& +\varepsilon(1-\varepsilon \alpha)\|n\|_{0}^{2}+\frac{\varepsilon^{3}}{\lambda^{2}}\|n\|_{-1}^{2}+2 \gamma\left\|E_{x}\right\|_{0}^{2} \\
& +(2 \gamma+\varepsilon) \int_{D} n|E|^{2} d x \\
& \leq \frac{1}{2} \frac{d}{d t}\left[\frac{1}{\lambda^{2}}\|m\|_{-1}^{2}+(1-\varepsilon \alpha)\|n\|_{0}^{2}+\frac{\varepsilon^{2}}{\lambda^{2}}\|n\|_{-1}^{2}\right] \\
& +\frac{d}{d t}\left(\left\|E_{x}\right\|_{0}^{2}+\int_{D} n|E|^{2} d x\right)+\left(\alpha \lambda_{1}-\frac{\varepsilon}{\lambda^{2}}\right)\|m\|_{-1}^{2} \\
& +\varepsilon(1-\varepsilon \alpha)\|n\|_{0}^{2}+\frac{\varepsilon^{3}}{\lambda^{2}}\|n\|_{-1}^{2}+2 \gamma\left\|E_{x}\right\|_{0}^{2} \\
& +(2 \gamma+\varepsilon) \int_{D} n|E|^{2} d x \leq \int_{D} f(-\Delta)^{-1} m d x \\
& +\int_{D} \dot{W}_{1}(-\Delta)^{-1} m d x+\frac{\lambda^{2}}{2}\left\|q_{1}\right\|_{-1}^{2}+\left\|q_{2}\right\|_{1}^{2} \\
& -2 \operatorname{Re} \int_{D}\left(\gamma \bar{E}+\bar{E}_{t}\right) g d x-2 \operatorname{Re} \int_{D}\left(\gamma \bar{E}+\bar{E}_{t}\right) \dot{W}_{2} d x \\
& +\int_{D} n\left|q_{2}\right|^{2} \mathrm{~d} x
\end{aligned}
$$

By Hölder inequality and Young inequality, we have the following estimates: 
$-2 \operatorname{Re} \int_{D}\left(\gamma \bar{E}+\bar{E}_{t}\right) g d x \leq 2\left\|E_{x}\right\|_{0}\left\|g_{x}\right\|_{0}+2\|g\|_{L^{\infty}}\|n\|_{0}\|E\|_{0}$

$$
\begin{aligned}
& -2 \operatorname{Re} \int_{D} i \dot{W}_{2} \bar{g} d x \leq \frac{\gamma}{2}\left\|E_{x}\right\|_{0}^{2} \\
& +\frac{2}{\gamma}\left\|g_{x}\right\|_{0}^{2}+C_{1}\left\|g_{x}\right\|_{0}^{4} \\
& +\frac{\varepsilon(1-\varepsilon \alpha)}{6}\|n\|_{0}^{2}+C_{2}\|E\|_{0}^{4} \\
& +2 \operatorname{Im} \int_{D} \dot{W}_{2} \bar{g} d x \\
& \left.(\gamma+\varepsilon)\left|\int_{D} n\right| E\right|^{2} d x \mid \leq C\|n\|_{0}\|E\|_{L^{4}}^{2} \leq C\|n\|_{0}\left(\|E\|_{0}^{3 / 2}\left\|E_{x}\right\|_{0}^{1 / 2}\right) \\
& \leq \frac{\varepsilon(1-\varepsilon \alpha)}{6}\|n\|_{0}^{2}+\frac{\gamma}{2}\left\|E_{x}\right\|_{0}^{2}+C_{3}\|E\|_{0}^{6}, \\
& \int_{D} f(-\Delta)^{-1} m d x \leq\|f\|_{-1}\|m\|_{-1} \\
& \leq \frac{\varepsilon}{2 \lambda^{2}}\|m\|_{-1}^{2}+\frac{\lambda^{2}}{2 \varepsilon}\|f\|_{-1}^{2}, \\
& \int_{D} n\left|q_{2}\right|^{2} d x \leq\|n\|_{0}\left\|q_{2}\right\|_{L^{4}}^{2} \leq\|n\|_{0}\left(C\left\|q_{2}\right\|_{0}^{3 / 2}\left\|q_{2 x}\right\|_{0}^{1 / 2}\right) \\
& \leq \frac{\varepsilon(1-\varepsilon \alpha)}{6}\|n\|_{0}^{2}+C\left(\left\|q_{2 x}\right\|_{0}^{2}+\left\|q_{2}\right\|_{0}^{6}\right) .
\end{aligned}
$$

By (38), we obtain

$$
\begin{aligned}
& \frac{d}{\mathrm{~d} t}\left[\frac{1}{2 \lambda^{2}}\|m\|_{-1}^{2}+\frac{1}{2}(1-\varepsilon \alpha)\|n\|_{0}^{2}+\frac{\varepsilon^{2}}{2 \lambda^{2}}\|n\|_{-1}^{2}\right] \\
& \quad+\frac{d}{d t}\left(\left\|E_{x}\right\|_{0}^{2}+\int_{D} n|E|^{2} d x\right)+\frac{\varepsilon}{\lambda^{2}}\|m\|_{-1}^{2} \\
& \quad+\varepsilon(1-\varepsilon \alpha)\|n\|_{0}^{2}+\frac{\varepsilon^{3}}{\lambda^{2}}\|n\|_{-1}^{2}+2 \gamma\left\|E_{x}\right\|_{0}^{2} \\
& \quad+(2 \gamma+\varepsilon) \int_{D} n|E|^{2} d x \\
& \leq \int_{D} f(-\Delta)^{-1} m d x+\int_{D} \dot{W}_{1}(-\Delta)^{-1} m d x \\
& \quad+\frac{\lambda^{2}}{2}\left\|q_{1}\right\|_{-1}^{2}+\left\|q_{2}\right\|_{1}^{2}-2 \operatorname{Re} \int_{D}\left(\gamma \bar{E}+\bar{E}_{t}\right) g d x \\
& \quad-2 \operatorname{Re} \int_{D}\left(\gamma \bar{E}+\bar{E}_{t}\right) \dot{W}_{2} d x+\int_{D} n\left|q_{2}\right|^{2} d x \\
& \leq \frac{\varepsilon}{2 \lambda^{2}}\|m\|_{-1}^{2}+\frac{\lambda^{2}}{2 \varepsilon}\|f\|_{-1}^{2}+\int_{D} \dot{W}_{1}(-\Delta)^{-1} m d x \\
&+\frac{\lambda^{2}}{2}\left\|q_{1}\right\|_{-1}^{2}+\left\|q_{2}\right\|_{1}^{2}+\frac{\gamma}{2}\left\|E_{x}\right\|_{0}^{2}+\frac{2}{\gamma}\left\|g_{x}\right\|_{0}^{2} \\
&+C_{1}\left\|g_{x}\right\|_{0}^{4}+\frac{\varepsilon(1-\varepsilon \alpha)}{6}\|n\|_{0}^{2}+C_{2}\|E\|_{0}^{4} \\
&+2 \operatorname{Im} \int_{D} \dot{W}_{2} \bar{g} d x-2 \operatorname{Re} \int_{D}\left(\gamma \bar{E}+\bar{E}_{t}\right) \dot{W_{2}} d x \\
&+\frac{\varepsilon(1-\varepsilon \alpha)}{6}\|n\|_{0}^{2}+C\left(\left\|q_{2}\right\|_{1}^{2}+\left\|q_{2}\right\|_{0}^{6}\right) .
\end{aligned}
$$

By (39), we get

$$
\begin{aligned}
\frac{d}{d t}\left[\frac{1}{2 \lambda^{2}}\|m\|_{-1}^{2}+\frac{1}{2}(1-\varepsilon \alpha)\|n\|_{0}^{2}+\frac{\varepsilon^{2}}{2 \lambda^{2}}\|n\|_{-1}^{2}\right] \\
\quad+\frac{d}{d t}\left(\left\|E_{x}\right\|_{0}^{2}+\int_{D} n|E|^{2} d x\right)+\frac{\varepsilon}{\lambda^{2}}\|m\|_{-1}^{2} \\
\quad+\varepsilon(1-\varepsilon \alpha)\|n\|_{0}^{2}+\frac{\varepsilon^{3}}{\lambda^{2}}\|n\|_{-1}^{2}+2 \gamma\left\|E_{x}\right\|_{0}^{2}+\gamma \int_{D} n|E|^{2} d x \\
\leq \frac{\varepsilon}{2 \lambda^{2}}\|m\|_{-1}^{2}+\frac{\lambda^{2}}{2 \varepsilon}\|f\|_{-1}^{2}+\int_{D} \dot{W}_{1}(-\Delta)^{-1} m d x \\
\quad+\frac{\lambda^{2}}{2}\left\|q_{1}\right\|_{-1}^{2}+\left\|q_{2}\right\|_{1}^{2}+\frac{\gamma}{2}\left\|E_{x}\right\|_{0}^{2}+\frac{2}{\gamma}\left\|g_{x}\right\|_{0}^{2} \\
\quad+\frac{\varepsilon(1-\varepsilon \alpha)}{6}\|n\|_{0}^{2}+C_{2}\|E\|_{0}^{4}+C_{1}\left\|g_{x}\right\|_{0}^{4} \\
\quad+2 \operatorname{Im} \int_{D} \dot{W}_{2} \bar{g} d x-2 \operatorname{Re} \int_{D}\left(\gamma \bar{E}+\bar{E}_{t}\right) \dot{W}_{2} d x \\
\quad+\frac{\varepsilon(1-\varepsilon \alpha)}{6}\|n\|_{0}^{2}+C\left(\left\|q_{2}\right\|_{1}^{2}+\left\|q_{2}\right\|_{0}^{6}\right) \\
\quad+\frac{\varepsilon(1-\varepsilon \alpha)}{6}\|n\|_{0}^{2}+\frac{\gamma}{2}\left\|E_{x}\right\|_{0}^{2}+C_{3}\|E\|_{0}^{6} .
\end{aligned}
$$

Now, taking $\beta=\min \{\varepsilon, \gamma\}$ and letting $H_{0}(t)=\left(1 / 2 \lambda^{2}\right)$ $\|m\|_{-1}^{2}+((1-\varepsilon \alpha) / 2)\|n\|_{0}^{2}+\left(\varepsilon^{2} / 2 \lambda^{2}\right)\|n\|_{-1}^{2}+\left\|E_{x}\right\|_{0}^{2}+\int_{D} n$ $|E|^{2} d x$, the above inequality is changed into

$$
\begin{aligned}
\frac{d}{d t} H_{0}(t)+\beta H_{0}(t) \leq & \frac{d}{d t}\left[\frac{1}{2 \lambda^{2}}\|m\|_{-1}^{2}+\frac{1}{2}(1-\varepsilon \alpha)\|n\|_{0}^{2}\right. \\
& \left.+\frac{\varepsilon^{2}}{2 \lambda^{2}}\|n\|_{-1}^{2}\right]+\frac{d}{d t}\left(\left\|E_{x}\right\|_{0}^{2}\right. \\
& \left.+\int_{D} n|E|^{2} d x\right)+\frac{\varepsilon}{2 \lambda^{2}}\|m\|_{-1}^{2} \\
& +\frac{\varepsilon(1-\varepsilon \alpha)}{2}\|n\|_{0}^{2}+\frac{\varepsilon^{3}}{\lambda^{2}}\|n\|_{-1}^{2} \\
& +\gamma\left\|E_{x}\right\|_{0}^{2}+\gamma \int_{D} n|E|^{2} d x \\
\leq & C\left(\|f\|_{-1},\|g\|_{1},\left\|q_{1}\right\|_{-1},\left\|q_{2}\right\|_{1}\right) \\
& +\int_{D} \dot{W}_{1}(-\Delta)^{-1} m d x+C\|E\|_{0}^{6} \\
& +2 \operatorname{Im} \int_{D} \dot{W}_{2} \bar{g} d x \\
& -2 \operatorname{Re} \int_{D}\left(\gamma \bar{E}+\bar{E}_{t}\right) \dot{W}_{2} d x \\
\leq & C\left(f, g, q_{1}, q_{2}\right)+\int_{D} \dot{W}_{1}(-\Delta)^{-1} m d x \\
& +C\|E\|_{0}^{6}+2 \operatorname{Im} \int_{D} \dot{W}_{2} \bar{g} d x \\
& -2 \operatorname{Re} \int_{D}\left(\gamma \bar{E}+\bar{E}_{t}\right) \dot{W}_{2} d x .
\end{aligned}
$$


Integrating (41) from 0 to $t$, we obtain

$$
\begin{aligned}
H_{0}(t) & -H_{0}(0)+\beta \int_{0}^{t} H_{0}(s) d s \\
\leq & C \int_{0}^{t}\|E(s)\|_{0}^{6} d s+C\left(f, g, q_{1}, q_{2}\right) t \\
& +\int_{0}^{t} \int_{D} \dot{W}_{1}(-\Delta)^{-1} m d x d s+2 \int_{0}^{t} \operatorname{Im} \int_{D} \dot{W}_{2} \bar{g} d x d s \\
& -2 \int_{0}^{t} \operatorname{Re} \int_{D}\left(\gamma \bar{E}+\bar{E}_{t}\right) \dot{W}_{2} d x d s .
\end{aligned}
$$

Taking expectation on both sides of the above inequality, by (28), we get

$$
\begin{aligned}
& \mathbb{E} H_{0}(t)-\mathbb{E} H_{0}(0)+\beta \mathbb{E} \int_{0}^{t} H_{0}(s) d s \\
& \leq C \mathbb{E} \int_{0}^{t}\|E(s)\|_{0}^{6} d s+C\left(f, g, q_{1}, q_{2}\right) t \\
& \quad \leq C\left(E_{0}, f, g, q_{1}, q_{2}\right) t .
\end{aligned}
$$

By (23) and Gronwall inequality, we have

$$
\begin{aligned}
\mathbb{E} H_{0}(t) & \leq e^{-\beta t} \mathbb{E} H_{0}(0)+C\left(E_{0}, f, g, q_{1}, q_{2}\right) \\
& \leq C\left(E_{0}, f, g, q_{1}, q_{2}\right),
\end{aligned}
$$

where $C\left(E_{0}, f, g, q_{1}, q_{2}\right)$ is independent of $T$.

Since

$$
\begin{aligned}
\left.\left|\int_{D} n\right| E\right|^{2} d x \mid & \leq C\|n\|_{0}\|E\|_{L^{4}}^{2} \leq C\|n\|_{0}\left(\|E\|_{0}^{3 / 2}\left\|E_{x}\right\|_{0}^{1 / 2}\right) \\
& \leq \frac{1-\varepsilon \alpha}{4}\|n\|_{0}^{2}+\frac{1}{2}\left\|E_{x}\right\|_{0}^{2}+C\|E\|_{0}^{6},
\end{aligned}
$$

we have

$$
\begin{aligned}
& C\left(\|m\|_{-1}^{2}+\|n\|_{0}^{2}+\|n\|_{-1}^{2}+\left\|E_{x}\right\|_{0}^{2}\right)-C\|E\|_{0}^{6} \\
& \quad \leq H_{0}(t) \leq C\left(\|m\|_{-1}^{2}+\|n\|_{0}^{2}+\|n\|_{-1}^{2}+\left\|E_{x}\right\|_{0}^{2}\right)+C\|E\|_{0}^{6} .
\end{aligned}
$$

By (41) and (46), we have

$$
\begin{aligned}
\frac{d}{d t} H_{0}(t) \leq & C\left(f, g, q_{1}, q_{2}\right)+\int_{D} \dot{W}_{1}(-\Delta)^{-1} m d x \\
& +C\|E\|_{0}^{6}+2 \operatorname{Im} \int_{D} \dot{W}_{2} \bar{g} d x \\
& -2 \operatorname{Re} \int_{D}\left(\gamma \bar{E}+\bar{E}_{t}\right) \dot{W}_{2} d x
\end{aligned}
$$

Therefore, by (28) and (44), we have

$$
\begin{aligned}
\mathbb{E}( & \left.\|m\|_{-1}^{2}+\|n\|_{0}^{2}+\|n\|_{-1}^{2}+\left\|E_{x}\right\|_{0}^{2}\right) \\
\leq & C\left(\mathbb{E} H_{0}(t)+C \mathbb{E}\|E\|_{0}^{6}\right) \\
\leq & C\left[e^{-\beta t} \mathbb{E} H_{0}(0)+C\left(E_{0}, f, g, q_{1}, q_{2}\right)\right] \\
& +C\left[e^{-3 \gamma t / 2} \mathbb{E}\left\|E_{0}\right\|_{0}^{6}+C\left(\|g\|_{0},\left\|q_{2}\right\|_{0}\right)\right] \\
\leq & C\left(E_{0}, f, g, q_{1}, q_{2}\right),
\end{aligned}
$$

where $C\left(E_{0}, f, g, q_{1}, q_{2}\right)$ is independent of $T$.

By $(48)$, we obtain $\left(n_{t}, n, E\right) \in L^{\infty}\left(0, \infty ; L^{2}\left(\Omega, \mathscr{E}_{0}\right)\right)$.

Further, we give an estimate of $H_{0}^{p}(t)$ for any $p \geq 1$. First as

$$
\begin{aligned}
\|n E\|_{-1}^{2} & =\int_{D}(-\Delta)^{-1}(n E) n \bar{E} d x \leq\left\|(-\Delta)^{-1} n E\right\|_{L^{\infty}}\|n\|_{0}\|E\|_{0} \\
& \leq C\left\|(-\Delta)^{-1} n E\right\|_{1}\|n\|_{0}\|E\|_{0} \leq C\|n E\|_{-1}\|n\|_{0}\|E\|_{0}
\end{aligned}
$$

by (47), we have

$$
\|n E\|_{-1}^{2} \leq C\|n\|_{0}^{2}\|E\|_{0}^{2} \leq C\left(H_{0}(t)+\|E\|_{0}^{6}\right)\|E\|_{0}^{2}
$$

$$
\|m\|_{-1}^{2}+\|n\|_{0}^{2}+\|n\|_{-1}^{2}+\left\|E_{x}\right\|_{0}^{2} \leq C\left(H_{0}(t)+\|E\|_{0}^{6}\right) .
$$

Applying Itô's formula to $H_{0}^{p}(t)$, by (41), (50), (51), and Young inequality, we obtain

$$
\begin{aligned}
\frac{d}{d t} H_{0}^{p}(t)= & p H_{0}^{p-1}(t) \frac{d}{d t} H_{0}(t)+\frac{1}{2} p(p-1) C H_{0}^{p-2}(t) \\
& \cdot\left(\|E\|_{1}^{2}\left\|q_{2}\right\|_{1}^{2}+\|g\|_{0}^{2}\left\|q_{2}\right\|_{0}^{2}\right)+\frac{1}{2} p(p-1) C H_{0}^{p-2}(t) \\
& \cdot\left(\|n E\|_{-1}^{2}\left\|q_{2}\right\|_{1}^{2}+\|m\|_{-1}^{2}\left\|q_{2}\right\|_{-1}^{2}\right) \\
\leq & p H_{0}^{p-1}(t)\left[-\beta H_{0}(t)+C\left(f, g, q_{1}, q_{2}\right)+C\|E\|_{0}^{6}\right] \\
& +p H_{0}^{p-1}(t)\left[\int_{D} \dot{W}_{1}(-\Delta)^{-1} m d x+2 \operatorname{Im} \int_{D} \dot{W}_{2} \bar{g} d x\right] \\
& +p H_{0}^{p-1}(t)\left[-2 \operatorname{Re} \int_{D}\left(\gamma \bar{E}+\bar{E}_{t}\right) \dot{W}_{2} d x\right] \\
& +\frac{1}{2} p(p-1) C H_{0}^{p-2}(t)\left(\|E\|_{1}^{2}\left\|q_{2}\right\|_{1}^{2}+\|g\|_{0}^{2}\left\|q_{2}\right\|_{0}^{2}\right) \\
& +\frac{1}{2} p(p-1) C H_{0}^{p-2}(t)\left(\|n E\|_{-1}^{2}\left\|q_{2}\right\|_{1}^{2}\right) \\
& +\frac{1}{2} p(p-1) C H_{0}^{p-2}(t)\left(\|m\|_{-1}^{2}\left\|q_{2}\right\|_{-1}^{2}\right)
\end{aligned}
$$




$$
\begin{aligned}
\leq & -\beta p H_{0}^{p}(t)+p H_{0}^{p-1}(t)\left[C\left(f, g, q_{1}, q_{2}\right)+C\|E\|_{0}^{6}\right] \\
& +p H_{0}^{p-1}(t)\left[\int_{D} \dot{W}_{1}(-\Delta)^{-1} m d x+2 \operatorname{Im} \int_{D} \dot{W}_{2} \bar{g} d x\right] \\
& +p H_{0}^{p-1}(t)\left[-2 \operatorname{Re} \int_{D}\left(\gamma \bar{E}+\bar{E}_{t}\right) \dot{W}_{2} d x\right] \\
& +\frac{1}{2} p(p-1) C H_{0}^{p-2}(t)\left[2\left(H_{0}(t)+C\|E\|_{0}^{6}\right)\right]\left\|q_{2}\right\|_{1}^{2} \\
& +\frac{1}{2} p(p-1) C H_{0}^{p-2}(t)\|g\|_{0}^{2}\left\|q_{2}\right\|_{0}^{2} \\
& +\frac{1}{2} p(p-1) C H_{0}^{p-2}(t)\left[\left(H_{0}(t)+\|E\|_{0}^{6}\right) C\|E\|_{0}^{2}\right]\left\|q_{2}\right\|_{1}^{2} \\
& +\frac{1}{2} p(p-1) C H_{0}^{p-2}(t)\left[2 \lambda^{2}\left(H_{0}(t)+C\|E\|_{0}^{6}\right)\right]\left\|q_{2}\right\|_{-1}^{2} \\
\leq & -\frac{\beta p}{2} H_{0}^{p}(t)+C\left(E_{0}, f, g, q_{1}, q_{2}\right)+C\left(\|E\|_{0}^{6 p}+\|E\|_{0}^{3 p}\right) \\
& +p H_{0}^{p-1}(t)\left[\int_{D} \dot{W}_{1}(-\Delta)^{-1} m d x+2 \operatorname{Im} \int_{D} \dot{W}_{2} \bar{g} d x\right] \\
& +p H_{0}^{p-1}(t)\left[-2 \operatorname{Re} \int_{D}\left(\gamma \bar{E}+\bar{E}_{t}\right) \dot{W}_{2} d x\right] .
\end{aligned}
$$

Integrating from 0 to $t$ and taking expectation on both sides of the above inequality, by Hölder inequality and (28), we derive

$$
\begin{gathered}
\mathbb{E} H_{0}^{p}(t) \leq \mathbb{E} H_{0}^{p}(0)-\frac{\beta p}{2} \mathbb{E} \int_{0}^{t} H_{0}^{p}(s) d s \\
+C\left(E_{0}, f, g, q_{1}, q_{2}\right) t .
\end{gathered}
$$

By Gronwall inequality, we have

$$
\begin{aligned}
\mathbb{E} H_{0}^{p}(t) & \leq e^{-\beta p t / 2} \mathbb{E} H_{0}^{p}(0)+C\left(E_{0}, f, g, q_{1}, q_{2}\right) \\
& \leq C\left(E_{0}, f, g, q_{1}, q_{2}, n_{0}, n_{1}\right),
\end{aligned}
$$

where $C\left(E_{0}, f, g, q_{1}, q_{2}, n_{0}, n_{1}\right)$ is independent of $T$.

By (28), (51), and (54), we obtain $\left(n_{t}, n, E\right) \in L^{\infty}(0, \infty$; $\left.L^{2 p}\left(\Omega, \mathscr{E}_{0}\right)\right)$.

On the other hand, integrating from 0 to $t$ on both sides of (47), deduce

$$
\begin{aligned}
H_{0}(t) \leq & H_{0}(0)+C\left(f, g, q_{1}, q_{2}\right) t \\
& +\left|\int_{0}^{t} \int_{D} \dot{W}_{1}(-\Delta)^{-1} m d x d s\right|^{2} \\
& +C \int_{0}^{t}\|E\|_{0}^{6} d s+\left|\int_{0}^{t} \operatorname{Im} \int_{D} \dot{W}_{2} g d x d s\right|^{2} \\
& +\left|\int_{0}^{t} \operatorname{Re} \int_{D}\left(\gamma \bar{E}+\bar{E}_{t}\right) \dot{W}_{2} d x d s\right|^{2}+C .
\end{aligned}
$$

Now, taking the supremum and expectation on both sides of the above inequality, we have

$$
\begin{aligned}
& \mathbb{E} \sup _{0 \leq t \leq T} H_{0}(t) \leq \mathbb{E} H_{0}(0)+C\left(f, g, q_{1}, q_{2}\right) T+C \\
& +\mathbb{E} \sup _{0 \leq t \leq T}\left|\int_{0}^{t} \int_{D} \dot{W}_{1}(-\Delta)^{-1} m d x d s\right|^{2} \\
& +C \mathbb{E} \sup _{0 \leq t \leq T} \int_{0}^{t}\|E\|_{0}^{6} d s \\
& +\mathbb{E} \sup _{0 \leq t \leq T}\left|\int_{0}^{t} \operatorname{Im} \int_{D} \dot{W}_{2} g d x d s\right|^{2} \\
& +\mathbb{E} \sup _{0 \leq t \leq T}\left|\int_{0}^{t} \operatorname{Re} \int_{D}\left(\gamma \bar{E}+\bar{E}_{t}\right) \dot{W}_{2} d x d s\right|^{2} \\
& \leq \mathbb{E} H_{0}(0)+C\left(f, g, q_{1}, q_{2}\right) T \\
& +C\left\|q_{1}\right\|_{-1}^{2} \mathbb{E} \int_{0}^{T}\|m\|_{-1}^{2} d t+C \mathbb{E} \int_{0}^{T}\|E\|_{0}^{6} d s \\
& +C\left\|q_{2}\right\|_{0}^{2} \mathbb{E} \int_{0}^{T}\|g\|_{0}^{2} d t+C \\
& +\mathbb{E} \sup _{0 \leq t \leq T} \mid \int_{0}^{t} \operatorname{Re} \int_{D}\left(-i \bar{E}_{x x}+\operatorname{in} \bar{E}\right. \\
& \left.+i \bar{g}+i \bar{W}_{2}\right)\left.\dot{W}_{2} d x d s\right|^{2} \\
& \leq \mathbb{E} \mathrm{H}_{0}(0)+C\left(f, g, q_{1}, q_{2}\right) T \\
& +C\left\|q_{1}\right\|_{-1}^{2} \mathbb{E} \int_{0}^{T}\|m\|_{-1}^{2} d t+C \mathbb{E} \int_{0}^{T}\|E\|_{0}^{6} d s \\
& +C\left\|q_{2}\right\|_{0}^{2} \mathbb{E} \int_{0}^{T}\|g\|_{0}^{2} d t+C \\
& +C\left\|q_{2}\right\|_{1}^{2} \mathbb{E} \int_{0}^{T}\left\|E_{x}\right\|_{0}^{2} d t+C\left\|q_{2}\right\|_{1}^{2} \mathbb{E} \int_{0}^{T}\|n E\|_{-1}^{2} d t \\
& \leq \mathbb{E} H_{0}(0)+C\left(f, g, q_{1}, q_{2}\right) T+C \\
& +C \mathbb{E} \int_{0}^{T}\|E\|_{0}^{6} d s+C\left\|q_{2}\right\|_{0}^{2} \mathbb{E} \int_{0}^{T}\|g\|_{0}^{2} d t \\
& +C\left\|q_{1}\right\|_{-1}^{2} \mathbb{E} \int_{0}^{T} 2 \lambda^{2}\left[H_{0}(t)+C\|E\|_{0}^{6}\right] d t \\
& +C\left\|q_{2}\right\|_{1}^{2} \mathbb{E} \int_{0}^{T} 2\left[H_{0}(t)+C\|E\|_{0}^{6}\right] d t \\
& +C\left\|q_{2}\right\|_{1}^{2} \mathbb{E} \int_{0}^{T} C\left[H_{0}(t)+\|E\|_{0}^{6}\right]\|E\|_{0}^{2} d t .
\end{aligned}
$$

Then, by (28), (54), and (56), we obtain

$$
\mathbb{E} \sup _{0 \leq t \leq T} H_{0}(t) \leq C_{T}\left(E_{0}, f, g, q_{1}, q_{2}, n_{1}, n_{0}\right),
$$

where $C_{T}$ depends on the initial data.

Therefore, by (48), we have

$$
\mathbb{E} \sup _{0 \leq t \leq T}\left\|\left(n_{t}, n, E\right)\right\|_{\mathscr{E}_{0}}^{2} \leq C_{T}\left(E_{0}, f, g, q_{1}, q_{2}, n_{1}, n_{0}\right) .
$$

By (58), we obtain $E \in L^{2}\left(\Omega ; L^{\infty}\left(0, T ; \mathscr{E}_{0}\right)\right)$. 
In addition, from (52), we have

$$
\begin{aligned}
\frac{d}{d t} H_{0}^{p}(t) \leq & C\left(E_{0}, f, g, q_{1}, q_{2}\right)+C\left(\|E\|_{0}^{6 p}+\|E\|_{0}^{3 p}\right) \\
& +p H_{0}^{p-1}(t)\left[\int_{D} \dot{W}_{1}(-\Delta)^{-1} m d x\right. \\
& \left.+2 \operatorname{Im} \int_{D} \dot{W}_{2} \bar{g} d x\right]+p H_{0}^{p-1}(t) \\
& \cdot\left[-2 \operatorname{Re} \int_{D}\left(\gamma \bar{E}+\bar{E}_{t}\right) \dot{W}_{2} d x\right] .
\end{aligned}
$$

Integrating from 0 to $t$ and taking the supremum and the expectation on both sides of (59), we have

$$
\begin{aligned}
\mathbb{E} \sup _{0 \leq t \leq T} H_{0}^{p}(t) \leq & C\left(E_{0}, f, g, q_{1}, q_{2}\right) T \\
& +C \mathbb{E} \sup _{0 \leq t \leq T} \int_{0}^{t}\left(\|E\|_{0}^{6 p}+\|E\|_{0}^{3 p}\right) d s \\
& +p H_{0}^{p-1}(t) \int_{D} \dot{W}_{1}(-\Delta)^{-1} m d x \\
& +2 p H_{0}^{p-1}(t) \operatorname{Im} \int_{D} \dot{W}_{2} \bar{g} d x \\
& +p H_{0}^{p-1}(t)\left[-2 \operatorname{Re} \int_{D}\left(-i \bar{E}_{x x}\right.\right. \\
& \left.\left.+i n \bar{E}+i \bar{g}+i \dot{W}_{2}\right) \dot{W}_{2} d x\right] \\
\leq & C\left(E_{0}, f, g, q_{1}, q_{2}\right) T \\
& +\mathbb{E} \sup _{0 \leq t \leq T} \int_{0}^{t}\left(\|E\|_{0}^{6 p}+\|E\|_{0}^{3 p}\right) d s \\
& +\mathbb{E} \sup _{0 \leq t \leq T}\left|\int_{0}^{t} H_{0}^{p-1}(t) \int_{D} \dot{W}_{1}(-\Delta)^{-1} m d x d s\right|^{2} \\
& +\mathbb{E} \sup _{0 \leq t \leq T}\left|\int_{0}^{t} H_{0}^{p-1}(t) \operatorname{Im} \int_{D} \dot{W}_{2} \bar{g} d x d s\right|^{2} \\
& +C+\mathbb{E} \sup _{0 \leq t \leq T}\left|\int_{0}^{t} H_{0}^{p-1}(t) \operatorname{Im} \bar{E}_{D} \bar{E}_{x x} \dot{W}_{2} d x d s\right|^{2} \\
& +\mathbb{E} \sup _{0 \leq t \leq T}\left|\int_{0}^{t} H_{0}^{p-1}(t) \operatorname{Im} \int_{D} n \bar{E} \dot{W}_{2} d x d s\right|^{2} .
\end{aligned}
$$

By (50), (51), Hölder inequality, and Young inequality, we have the following estimates:

$$
\begin{gathered}
C \mathbb{E} \sup _{0 \leq t \leq T} \int_{0}^{t}\left(\|E\|_{0}^{6 p}+\|E\|_{0}^{3 p}\right) d s \leq C \mathbb{E} \int_{0}^{T}\left(\|E\|_{0}^{6 p}+\|E\|_{0}^{3 p}\right) d s, \\
\mathbb{E} \sup _{0 \leq t \leq T}\left|\int_{0}^{t} H_{0}^{p-1}(t) \int_{D} \dot{W}_{1}(-\Delta)^{-1} m d x d s\right|^{2} \\
\leq C\left\|q_{1}\right\|_{-1}^{2} \mathbb{E} \int_{0}^{T} H_{0}^{2 p-2}(t)\|m\|_{-1}^{2} d s
\end{gathered}
$$

$$
\begin{aligned}
& \leq C\left\|q_{1}\right\|_{-1}^{2} \mathbb{E} \int_{0}^{T} H_{0}^{2 p-2}(t)\left[C\left(H_{0}(t)+\|E\|_{0}^{6}\right)\right] d s \\
& \leq C\left\|q_{1}\right\|_{-1}^{2} \mathbb{E} \int_{0}^{T}\left[H_{0}^{2 p}(t)+\|E\|_{0}^{6 p}+1\right] d s, \\
& \mathbb{E} \sup _{0 \leq t \leq T}\left|\int_{0}^{t} H_{0}^{p-1}(t) \operatorname{Im} \int_{D} \dot{W}_{2} \bar{g} d x d s\right|^{2} \\
& \leq C\left\|q_{2}\right\|_{0}^{2} \mathbb{E} \int_{0}^{T} H_{0}^{2 p-2}(t)\|g\|_{0}^{2} d s \\
& \leq C\left\|q_{2}\right\|_{0}^{2} \mathbb{E} \int_{0}^{T}\left[H_{0}^{2 p}(t)+\|g\|_{0}^{2 p}\right] d s \\
& \leq C\left\|q_{2}\right\|_{0}^{2} \mathbb{E} \int_{0}^{T}\left[H_{0}^{2 p}(t)+1\right] d s, \\
& \mathbb{E} \sup _{0 \leq t \leq T}\left|\int_{0}^{t} H_{0}^{p-1}(t) \operatorname{Im} \int_{D} \bar{E}_{x x} \dot{W}_{2} d x d s\right|^{2} \\
& \leq C\left\|q_{2 x}\right\|_{0}^{2} \mathbb{E} \int_{0}^{T} H_{0}^{2 p-2}(t)\left\|E_{x}\right\|_{0}^{2} d s \\
& \leq C\left\|q_{2 x}\right\|_{0}^{2} \mathbb{E} \int_{0}^{T} H_{0}^{2 p-2}(t)\left[C\left(H_{0}(t)+\|E\|_{0}^{6}\right)\right] d s \\
& \leq C\left\|q_{2 x}\right\|_{0}^{2} \mathbb{E} \int_{0}^{T}\left[H_{0}^{2 p}(t)+\|E\|_{0}^{6 p}+1\right] d s, \\
& \underset{0 \leq s \leq T}{\mathbb{E} \sup _{0}}\left|\int_{0}^{t} H_{0}^{p-1}(t) \operatorname{Im} \int_{D} n \bar{E} \dot{W}_{2} d x d s\right|^{2} \\
& \leq C\left\|q_{2}\right\|_{1}^{2} \mathbb{E} \int_{0}^{T} H_{0}^{2 p-2}(t)\|n E\|_{-1}^{2} d s \\
& \leq C\left\|q_{2}\right\|_{1}^{2} \mathbb{E} \int_{0}^{T} H_{0}^{2 p-2}(t)\left[C\left(H_{0}(t)+\|E\|_{0}^{6}\right)\|E\|_{0}^{2}\right] d s \\
& \leq C\left\|q_{2}\right\|_{1}^{2} \mathbb{E} \int_{0}^{T}\left[H_{0}^{2 p}(t)+\|E\|_{0}^{4 p}+\|E\|_{0}^{8 p}\right] d s .
\end{aligned}
$$

By (28), (54), (60), and (61), we have

$$
\begin{aligned}
\mathbb{E} \sup _{0 \leq t \leq T} H_{0}^{p}(t) & \leq C \mathbb{E} H_{0}^{p}(0)+C_{T}\left(E_{0}, f, g, q_{1}, q_{2}, n_{1}, n_{0}\right) \\
& \leq C_{T}\left(E_{0}, f, g, q_{1}, q_{2}, n_{1}, n_{0}\right) .
\end{aligned}
$$

Moreover, by (29), (51), and (62), we have

$$
\begin{aligned}
C \mathbb{E} & \sup _{0 \leq t \leq T}\left(\|m\|_{-1}^{2 p}+\|n\|_{0}^{2 p}+\|n\|_{-1}^{2 p}+\left\|E_{x}\right\|_{0}^{2 p}\right) \\
& \leq C \mathbb{E} \sup _{0 \leq t \leq T}\left[C\left(H_{0}(t)+\|E\|_{0}^{6}\right)\right]^{2 p} \\
& \leq C \mathbb{E} \sup _{0 \leq t \leq T}\left[H_{0}^{2 p}(t)+\|E\|_{0}^{12 p}\right] \\
& \leq C_{T}\left(E_{0}, f, g, q_{1}, q_{2}, n_{1}, n_{0}\right),
\end{aligned}
$$

where $C_{T}$ depends on the initial data. 
Therefore, by (63), we have

$\mathbb{E} \sup _{0 \leq t \leq T}\left\|\left(n_{\mathrm{t}}, n, E\right)\right\|_{\mathscr{E}_{0}}^{2 p} \leq C_{T}\left(E_{0}, f, g, q_{1}, q_{2}, n_{1}, n_{0}\right)$.

By (64), we obtain $E \in L^{2 p}\left(\Omega ; L^{\infty}\left(0, T ; \mathscr{E}_{0}\right)\right)$.

Lemma 4 is proved completely.

Lemma 5. Assume $\left(n_{1}, n_{0}, E_{0}\right) \in \mathscr{E}_{1}, f \in L^{2}(D), q_{1} \in H_{0}^{1}(D)$, and $g, q_{2} \in H^{2}(D) \cap H_{0}^{1}(D)$. Then, for any $T>0$ and $p \geq 1$, $\left(n_{t}, n, E\right) \in L^{2 p}\left(\Omega ; L^{\infty}\left(0, T ; \mathscr{E}_{1}\right)\right) \cap L^{\infty}\left(0, \infty ; L^{2 p}\left(\Omega, \mathscr{E}_{1}\right)\right)$.

Proof. Applying Itô's formula to $\|m\|_{0}^{2}$, by (12), we have

$$
\begin{aligned}
\frac{1}{2 \lambda^{2}} \frac{d}{d t}\|m\|_{0}^{2}= & \int_{D} \frac{1}{\lambda^{2}} m_{t} m d x+\frac{\lambda^{2}}{2}\left\|q_{1}\right\|_{0}^{2} \\
= & \frac{\varepsilon}{\lambda^{2}}\|m\|_{0}^{2}-\alpha\|m\|_{1}^{2}-\frac{\varepsilon^{2}}{\lambda^{2}} \int_{D} n m d x \\
& +(1-\varepsilon \alpha) \int_{D} n_{x x} m d x+\int_{D}|E|_{x x}^{2} m d x \\
& +\int_{D} f m d x+\int_{D} \dot{W}_{1} m d x+\frac{\lambda^{2}}{2}\left\|q_{1}\right\|_{0}^{2} .
\end{aligned}
$$

Since $m=n_{t}+\varepsilon n$, from (65), we obtain

$$
\begin{aligned}
& \frac{d}{d t}\left(\frac{1}{2 \lambda^{2}}\|m\|_{0}^{2}+\frac{\varepsilon^{2}}{2 \lambda^{2}}\|n\|_{0}^{2}+\frac{1-\varepsilon \alpha}{2}\left\|n_{x}\right\|_{0}^{2}\right) \\
& =\frac{\varepsilon}{\lambda^{2}}\|m\|_{0}^{2}-\alpha\|m\|_{1}^{2}-\frac{\varepsilon^{3}}{\lambda^{2}}\|n\|_{0}^{2}-\varepsilon(1-\varepsilon \alpha)\left\|n_{x}\right\|_{0}^{2} \\
& \quad+\int_{D}|E|_{x x}^{2} m d x+\int_{D} f m d x+\int_{D} \dot{W}_{1} m d x+\frac{\lambda^{2}}{2}\left\|q_{1}\right\|_{0}^{2} \\
& \leq\left(\frac{\varepsilon}{\lambda^{2}}-\alpha \lambda_{1}\right)\|m\|_{0}^{2}-\frac{\varepsilon^{3}}{\lambda^{2}}\|n\|_{0}^{2}-\varepsilon(1-\varepsilon \alpha)\left\|n_{x}\right\|_{0}^{2} \\
& \quad+\int_{D}|E|_{x x}^{2} m d x+\int_{D} f m d x+\int_{D} \dot{W}_{1} m d x+\frac{\lambda^{2}}{2}\left\|q_{1}\right\|_{0}^{2},
\end{aligned}
$$

where $\lambda_{1}$ is the first eigenvalue of $-\Delta$. By (66), we obtain

$$
\begin{aligned}
& \frac{d}{d t}\left(\frac{1}{2 \lambda^{2}}\|m\|_{0}^{2}+\frac{\varepsilon^{2}}{2 \lambda^{2}}\|n\|_{0}^{2}+\frac{1-\varepsilon \alpha}{2}\left\|n_{x}\right\|_{0}^{2}\right) \\
& \quad-\left(\frac{\varepsilon}{\lambda^{2}}-\alpha \lambda_{1}\right)\|m\|_{0}^{2}+\frac{\varepsilon^{3}}{\lambda^{2}}\|n\|_{0}^{2}+\varepsilon(1-\varepsilon \alpha)\left\|n_{x}\right\|_{0}^{2} \\
& \quad \leq \int_{D}|E|_{x x}^{2} m d x+\int_{D} f m d x+\int_{D} \dot{W}_{1} m d x+\frac{\lambda^{2}}{2}\left\|q_{1}\right\|_{0}^{2} .
\end{aligned}
$$

Applying Itô's formula to $\left\|E_{x x}\right\|_{0}^{2}$, by (13), we have

$$
\frac{d}{d t}\left\|E_{x x}\right\|_{0}^{2}=2 \operatorname{Re} \int_{D} E_{x x} \bar{E}_{t x x} d x+\left\|q_{2}\right\|_{2}^{2}
$$

Substituting $E_{x x}=-i E_{t}+n E-i \gamma E+g+\dot{W}_{2}$ into (68), we have

$$
\begin{aligned}
\frac{d}{d t}\left\|E_{x x}\right\|_{0}^{2}= & 2 \operatorname{Re} \int_{D}\left(-i E_{t}+n E-i \gamma E+g+\dot{W}_{2}\right) \bar{E}_{t x x} d x \\
& +\left\|q_{2}\right\|_{2}^{2}=2 \operatorname{Re} \int_{D} n E \bar{E}_{t x x} d x \\
& +2 \operatorname{Re} \int_{D} g \bar{E}_{t x x} d x+2 \operatorname{Re} \int_{D} \dot{W}_{2} \bar{E}_{t x x} d x \\
& +\left\|q_{2}\right\|_{2}^{2}+2 \operatorname{Re} \int_{D}\left(\gamma \bar{E}_{x x}\right) \\
& +\left(-E_{x x}+n E-i \gamma E+g+\dot{W}_{2}\right) d x \\
= & 2 \operatorname{Re} \int_{D} n E\left(\bar{E}_{t x x}+\gamma \bar{E}_{x x}\right) d x \\
& +2 \operatorname{Re} \int_{D} g\left(\bar{E}_{t x x}+\gamma \bar{E}_{x x}\right) d x \\
& +2 \operatorname{Re} \int_{D} \dot{W}_{2}\left(\bar{E}_{t x x}+\gamma \bar{E}_{x x}\right) d x \\
& -2 \gamma\left\|E_{x x}\right\|_{0}^{2}+\left\|q_{2}\right\|_{2}^{2} .
\end{aligned}
$$

From (66) and (69), we obtain

$$
\begin{aligned}
& \frac{d}{d t}\left(\frac{1}{2 \lambda^{2}}\|m\|_{0}^{2}+\frac{\varepsilon^{2}}{2 \lambda^{2}}\|n\|_{0}^{2}+\frac{1-\varepsilon \alpha}{2}\left\|n_{x}\right\|_{0}^{2}+\left\|E_{x x}\right\|_{0}^{2}\right) \\
& \quad-\left(\frac{\varepsilon}{\lambda^{2}}-\alpha \lambda_{1}\right)\|m\|_{0}^{2}+\frac{\varepsilon^{3}}{\lambda^{2}}\|n\|_{0}^{2}+\varepsilon(1-\varepsilon \alpha)\left\|n_{x}\right\|_{0}^{2} \\
& \leq \int_{D}|E|_{x x}^{2} m d x+\int_{D} f m d x+\int_{D} \dot{W}_{1} m d x+\frac{\lambda^{2}}{2}\left\|q_{1}\right\|_{0}^{2} \\
& \quad+2 \operatorname{Re} \int_{D} n E\left(\bar{E}_{t x x}+\gamma \bar{E}_{x x}\right) d x \\
& \quad+2 \operatorname{Re} \int_{D} g\left(\bar{E}_{t x x}+\gamma \bar{E}_{x x}\right) d x \\
& \quad+2 \operatorname{Re} \int_{D} \dot{W}_{2}\left(\bar{E}_{t x x}+\gamma \bar{E}_{x x}\right) d x-2 \gamma\left\|E_{x x}\right\|_{0}^{2}+\left\|q_{2}\right\|_{2}^{2},
\end{aligned}
$$

since

$$
\begin{aligned}
\frac{d}{d t}( & \left.-2 \operatorname{Re} \int_{D} n E \bar{E}_{x x} d x\right) \\
= & -\operatorname{Re} \int_{D} n_{t}|E|_{x x}^{2} d x+2 \operatorname{Re} \int_{D} n_{t}\left|E_{x}\right|^{2} d x \\
& -2 \operatorname{Re} \int_{D} n E \bar{E}_{t x x} d x-2 \operatorname{Re} \int_{D} n E_{t} \bar{E}_{x x} d x \\
& -2 \operatorname{Re} \int_{D} n q_{2} \bar{q}_{2 x x} d x .
\end{aligned}
$$


Substituting (71) into (70), we have

$$
\begin{aligned}
& \frac{d}{d t}\left(\frac{1}{2 \lambda^{2}}\|m\|_{0}^{2}+\frac{\varepsilon^{2}}{2 \lambda^{2}}\|n\|_{0}^{2}+\frac{1-\varepsilon \alpha}{2}\left\|n_{x}\right\|_{0}^{2}+\left\|E_{x x}\right\|_{0}^{2}\right) \\
& -\left(\frac{\varepsilon}{\lambda^{2}}-\alpha \lambda_{1}\right)\|m\|_{0}^{2}+\frac{\varepsilon^{3}}{\lambda^{2}}\|n\|_{0}^{2}+\varepsilon(1-\varepsilon \alpha)\left\|n_{x}\right\|_{0}^{2} \\
& \leq \int_{D}|E|_{x x}^{2} m d x+\int_{D} f m d x+\int_{D} \dot{W}_{1} m d x \\
& +\frac{\lambda^{2}}{2}\left\|q_{1}\right\|_{0}^{2}+2 \operatorname{Re} \int_{D} n E \bar{E}_{t x x} d x \\
& +2 \gamma \operatorname{Re} \int_{D} n E \bar{E}_{x x} d x+2 \operatorname{Re} \int_{D} g\left(\bar{E}_{t x x}+\gamma \bar{E}_{x x}\right) d x \\
& +2 \operatorname{Re} \int_{D} \dot{W}_{2}\left(\bar{E}_{t x x}+\gamma \bar{E}_{x x}\right) d x-2 \gamma\left\|E_{x x}\right\|_{0}^{2}+\left\|q_{2}\right\|_{2}^{2} \\
& \leq \int_{D}|E|_{x x}^{2} m d x+\int_{D} f m d x+\int_{D} \dot{W}_{1} m d x \\
& +\frac{\lambda^{2}}{2}\left\|q_{1}\right\|_{0}^{2}-\operatorname{Re} \int_{D} n_{t}|E|_{x x}^{2} d x+2 \operatorname{Re} \int_{D} n_{t}\left|E_{x}\right|^{2} d x \\
& +\frac{d}{d t}\left(2 \operatorname{Re} \int_{D} n E \bar{E}_{x x} d x\right)-2 \operatorname{Re} \int_{D} n E_{t} \bar{E}_{x x} d x \\
& -2 \operatorname{Re} \int_{D} n q_{2} \bar{q}_{2 x x} d x+2 \gamma \operatorname{Re} \int_{D} n E \bar{E}_{x x} d x \\
& +2 \operatorname{Re} \int_{D} g\left(\bar{E}_{t x x}+\gamma \bar{E}_{x x}\right) d x \\
& +2 \operatorname{Re} \int_{D} \dot{W}_{2}\left(\bar{E}_{t x x}+\gamma \bar{E}_{x x}\right) d x-2 \gamma\left\|E_{x x}\right\|_{0}^{2}+\left\|q_{2}\right\|_{2}^{2} \text {. }
\end{aligned}
$$
have

Taking $0<\varepsilon \leq \min \left\{1 / \alpha, \alpha \lambda_{1} \lambda^{2} / 2\right\}$, and by (72), we

$$
\begin{aligned}
& \frac{d}{d t}\left(\frac{1}{2 \lambda^{2}}\|m\|_{0}^{2}+\frac{\varepsilon^{2}}{2 \lambda^{2}}\|n\|_{0}^{2}+\frac{1-\varepsilon \alpha}{2}\left\|n_{x}\right\|_{0}^{2}\right) \\
& \quad+\frac{d}{d t}\left(\left\|E_{x x}\right\|_{0}^{2}-2 \operatorname{Re} \int_{D} n E \bar{E}_{x x} d x\right) \\
& \quad+\frac{\varepsilon}{\lambda^{2}}\|m\|_{0}^{2}+\frac{\varepsilon^{3}}{\lambda^{2}}\|n\|_{0}^{2}+\varepsilon(1-\varepsilon \alpha)\left\|n_{x}\right\|_{0}^{2} \\
& \quad+2 \gamma\left\|E_{x x}\right\|_{0}^{2}-2 \gamma \operatorname{Re} \int_{D} n E \bar{E}_{x x} d x \\
& \leq \int_{D}|E|_{x x}^{2} m d x+\int_{D} f m d x+\int_{D} \dot{W}_{1} m d x \\
& \quad+\frac{\lambda^{2}}{2}\left\|q_{1}\right\|_{0}^{2}-\operatorname{Re} \int_{D} n_{t}|E|_{x x}^{2} d x \\
& \quad+2 \operatorname{Re} \int_{D} n_{t}\left|E_{x}\right|^{2} d x+\left\|q_{2}\right\|_{2}^{2}-2 \operatorname{Re} \int_{D} n E_{t} \bar{E}_{x x} d x \\
& \quad-2 \operatorname{Re} \int_{D} n q_{2} \bar{q}_{2 x x} d x+2 \operatorname{Re} \int_{D} g\left(\bar{E}_{t x x}+\gamma \bar{E}_{x x}\right) d x \\
& \quad+2 \operatorname{Re} \int_{D} \dot{W}_{2}\left(\bar{E}_{t x x}+\gamma \bar{E}_{x x}\right) d x
\end{aligned}
$$

$$
\begin{aligned}
& \leq \int_{D} f m d x+\int_{D} \dot{W}_{1} m d x+\frac{\lambda^{2}}{2}\left\|q_{1}\right\|_{0}^{2} \\
& \quad-\varepsilon \operatorname{Re} \int_{D} n_{x}|E|_{x}^{2} d x+2 \operatorname{Re} \int_{D} m\left|E_{x}\right|^{2} d x \\
& \quad-2 \varepsilon \operatorname{Re} \int_{D} n\left|E_{x}\right|^{2} d x+\left\|q_{2}\right\|_{2}^{2} \\
& \quad-2 \operatorname{Re} \int_{D} n E_{t} \bar{E}_{x x} d x-2 \operatorname{Re} \int_{D} n q_{2} \bar{q}_{2 x x} d x \\
& \quad+2 \operatorname{Re} \int_{D} g\left(\bar{E}_{t x x}+\gamma \bar{E}_{x x}\right) d x \\
& +2 \operatorname{Re} \int_{D} \dot{W}_{2}\left(\bar{E}_{t x x}+\gamma \bar{E}_{x x}\right) d x .
\end{aligned}
$$

By Hölder inequality and Young inequality, we have the following estimates:

$$
\begin{aligned}
& \int_{D} f m d x \leq\|f\|_{0}\|m\|_{0} \leq C\|f\|_{0}^{2}+\frac{\varepsilon}{4 \lambda^{2}}\|m\|_{0}^{2}, \\
& -\varepsilon \operatorname{Re} \int_{D} n_{x}|E|_{x}^{2} d x+2 \operatorname{Re} \int_{D} m\left|E_{x}\right|^{2} d x-2 \varepsilon \operatorname{Re} \int_{D} n\left|E_{x}\right|^{2} d x \\
& \leq \varepsilon\left\|n_{x}\right\|_{0}\left\|E_{x}\right\|_{L^{4}}^{2}+2\|m\|_{0}\left\|E_{x}\right\|_{L^{4}}^{2}+2 \varepsilon\|n\|_{0}\left\|E_{x}\right\|_{L^{4}}^{2} \\
& \leq\left(\varepsilon\left\|n_{x}\right\|_{0}+2\|m\|_{0}+2 \varepsilon\|n\|_{0}\right)\left\|E_{x}\right\|_{0}^{3 / 2}\left\|E_{x x}\right\|_{0}^{1 / 2} \\
& \leq \frac{\varepsilon(1-\varepsilon \alpha)}{6}\left\|n_{x}\right\|_{0}^{2}+\frac{\varepsilon}{4 \lambda^{2}}\|m\|_{0}^{2}+\frac{\varepsilon^{3}}{3 \lambda^{2}}\|n\|_{0}^{2} \\
& +\frac{\gamma}{5}\left\|E_{x x}\right\|_{0}^{2}+C\left\|E_{x}\right\|_{0}^{6} \\
& -2 \operatorname{Re} \int_{D} n E_{t} \bar{E}_{x x} d x=2 \operatorname{Re} \int_{D}\left(\operatorname{in} \bar{E}_{x x}\right)\left(i E_{t}\right) d x \\
& =2 \operatorname{Re} \int_{D} i n^{2} \bar{E}_{x x} E d x+2 \gamma \operatorname{Re} \int_{D} n \bar{E}_{x x} E d x \\
& +2 \operatorname{Re} \int_{D} \operatorname{in} \bar{E}_{x x} g d x+2 \operatorname{Re} \int_{D} \operatorname{in} \bar{E}_{x x} \dot{W}_{2} d x \\
& \leq\|n\|_{L^{4}}^{2}\left\|E_{x x}\right\|_{0}\|E\|_{L^{\infty}}+\|n\|_{0}\left\|E_{x x}\right\|_{0}\|E\|_{L^{\infty}} \\
& +\|n\|_{0}\left\|E_{x x}\right\|_{0}\|g\|_{L^{\infty}}-2 \operatorname{Im} \int_{D} n \bar{E}_{x x} \dot{W}_{2} d x \\
& \leq\|n\|_{0}^{3 / 2}\left\|n_{x}\right\|_{0}^{1 / 2}\left\|E_{x x}\right\|_{0}\left\|E_{x}\right\|_{0}+\|n\|_{0}\left\|E_{x x}\right\|_{0}\left\|E_{x}\right\|_{0} \\
& +\|n\|_{0}\left\|E_{x x}\right\|_{0}\left\|g_{x}\right\|_{0}-2 \operatorname{Im} \int_{D} n \bar{E}_{x x} \dot{W}_{2} d x \\
& \leq C\|n\|_{0}^{12}+\frac{\varepsilon(1-\varepsilon \alpha)}{6}\left\|n_{x}\right\|_{0}^{2}+\frac{\gamma}{5}\left\|E_{x x}\right\|_{0}^{2} \\
& +C\left\|E_{x}\right\|_{0}^{8}+C\|n\|_{0}^{4}+\frac{2 \gamma}{5}\left\|E_{x x}\right\|_{0}^{2}+C\left\|E_{x}\right\|_{0}^{4} \\
& +C\left\|g_{x}\right\|_{0}^{4}-2 \operatorname{Im} \int_{D} n \bar{E}_{x x} \dot{W}_{2} d x \\
& \leq \frac{\varepsilon(1-\varepsilon \alpha)}{6}\left\|n_{x}\right\|_{0}^{2}+\frac{3 \gamma}{5}\left\|E_{x x}\right\|_{0}^{2}+C\left(\|n\|_{0}^{12}+\left\|E_{x}\right\|_{0}^{8}\right) \\
& +C\left(\|n\|_{0}^{4}+\left\|E_{x}\right\|_{0}^{4}+\left\|g_{x}\right\|_{0}^{4}\right)-2 \operatorname{Im} \int_{D} n \bar{E}_{x x} \dot{W}_{2} d x
\end{aligned}
$$


$-2 \operatorname{Re} \int_{D} n q_{2} \bar{q}_{2 x x} d x \leq \frac{\varepsilon^{3}}{3 \lambda^{2}}\|n\|_{0}^{2}+C\left\|q_{2 x x}\right\|_{0}^{4}$,

$2 \operatorname{Re} \int_{D}\left(\gamma \bar{E}_{x x}+\bar{E}_{t \times x}\right) g d x$

$$
\begin{aligned}
= & 2 \operatorname{Re} \int_{D}\left(-i \bar{E}_{x x}+\operatorname{in} \bar{E}+i \bar{g}+i \bar{W}_{2}\right)_{x x} g d x \\
\leq & 2\left\|E_{x x}\right\|_{0}\left\|g_{x x}\right\|_{0}+2\left\|g_{x}\right\|_{L^{\infty}}\left(\left\|n_{x}\right\|_{0}\|E\|_{0}+\|n\|_{0}\left\|E_{x}\right\|_{0}\right) \\
& -2 \operatorname{Re} \int_{D} i \dot{W}_{2} \bar{g}_{x x} d x \leq \frac{\gamma}{5}\left\|E_{x x}\right\|_{0}^{2}+C\left\|g_{x x}\right\|_{0}^{2}+C\left\|g_{x x}\right\|_{0}^{4} \\
& +\frac{\varepsilon(1-\varepsilon \alpha)}{6}\left\|n_{x}\right\|_{0}^{2}+C\|E\|_{0}^{4}+C\left\|g_{x x}\right\|_{0}^{4} \\
& +\frac{\varepsilon^{3}}{4 \lambda^{2}}\|n\|_{0}^{2}+C\left\|E_{x}\right\|_{0}^{4}+2 \operatorname{Im} \int_{D} \dot{W}_{2} \bar{g}_{x x} d x \\
\leq & \frac{\gamma}{5}\left\|E_{x x}\right\|_{0}^{2}+\frac{\varepsilon(1-\varepsilon \alpha)}{6}\left\|n_{x}\right\|_{0}^{2}+\frac{\varepsilon^{3}}{3 \lambda^{2}}\|n\|_{0}^{2} \\
& +2 \operatorname{Im} \int_{D} \dot{W}_{2} \bar{g}_{x x} d x \\
& +C\left(\left\|g_{x x}\right\|_{0}^{2}+\left\|g_{x x}\right\|_{0}^{4}+\|E\|_{0}^{4}+\left\|E_{x}\right\|_{0}^{4}\right) .
\end{aligned}
$$

By (73), we have

$$
\begin{aligned}
& \frac{d}{d t}\left(\frac{1}{2 \lambda^{2}}\|m\|_{0}^{2}+\frac{\varepsilon^{2}}{2 \lambda^{2}}\|n\|_{0}^{2}+\frac{1-\varepsilon \alpha}{2}\left\|n_{x}\right\|_{0}^{2}\right) \\
& +\frac{d}{d t}\left(\left\|E_{x x}\right\|_{0}^{2}-2 \operatorname{Re} \int_{D} n E \bar{E}_{x x} d x\right) \\
& +\frac{\varepsilon}{\lambda^{2}}\|m\|_{0}^{2}+\frac{\varepsilon^{3}}{\lambda^{2}}\|n\|_{0}^{2}+\varepsilon(1-\varepsilon \alpha)\left\|n_{x}\right\|_{0}^{2} \\
& +2 \gamma\left\|E_{x x}\right\|_{0}^{2}-2 \gamma \operatorname{Re} \int_{D} n E \bar{E}_{x x} d x \\
& \leq C\|f\|_{0}^{2}+\frac{\varepsilon}{4 \lambda^{2}}\|m\|_{0}^{2}+\int_{D} \dot{W}_{1} m d x+\frac{\lambda^{2}}{2}\left\|q_{1}\right\|_{0}^{2} \\
& +\frac{\varepsilon(1-\varepsilon \alpha)}{6}\left\|n_{x}\right\|_{0}^{2}+\frac{\varepsilon}{4 \lambda^{2}}\|m\|_{0}^{2}+\frac{\varepsilon^{3}}{6 \lambda^{2}}\|n\|_{0}^{2} \\
& +\frac{\gamma}{5}\left\|E_{x x}\right\|_{0}^{2}+C\left\|E_{x}\right\|_{0}^{6}+\left\|q_{2}\right\|_{2}^{2}+\frac{\varepsilon(1-\varepsilon \alpha)}{6}\left\|n_{x}\right\|_{0}^{2} \\
& +\frac{3 \gamma}{5}\left\|E_{x x}\right\|_{0}^{2}+C\left(\|n\|_{0}^{12}+\left\|E_{x}\right\|_{0}^{8}\right) \\
& +C\left(\|n\|_{0}^{4}+\left\|E_{x}\right\|_{0}^{4}+\left\|g_{x}\right\|_{0}^{4}\right)-2 \operatorname{Im} \int_{D} n \bar{E}_{x x} \dot{W}_{2} d x \\
& +\frac{\varepsilon^{3}}{6 \lambda^{2}}\|n\|_{0}^{2}+C\left\|q_{2 x x}\right\|_{0}^{4}+\frac{\gamma}{5}\left\|E_{x x}\right\|_{0}^{2} \\
& +\frac{\varepsilon(1-\varepsilon \alpha)}{6}\left\|n_{x}\right\|_{0}^{2}+\frac{\varepsilon^{3}}{6 \lambda^{2}}\|n\|_{0}^{2}+2 \operatorname{Im} \int_{D} \dot{W}_{2} \bar{g}_{x x} d x \\
& +C\left(\left\|g_{x x}\right\|_{0}^{2}+\left\|g_{x x}\right\|_{0}^{4}+\|E\|_{0}^{4}+\left\|E_{x}\right\|_{0}^{4}\right) \\
& +2 \operatorname{Re} \int_{D} \dot{W}_{2}\left(\bar{E}_{t x x}+\gamma \bar{E}_{x x}\right) d x \text {. }
\end{aligned}
$$

By (75), we get

$$
\begin{aligned}
& \frac{d}{d t}\left(\frac{1}{2 \lambda^{2}}\|m\|_{0}^{2}+\frac{\varepsilon^{2}}{2 \lambda^{2}}\|n\|_{0}^{2}+\frac{1-\varepsilon \alpha}{2}\left\|n_{x}\right\|_{0}^{2}\right) \\
& +\frac{d}{d t}\left(\left\|E_{x x}\right\|_{0}^{2}-2 \operatorname{Re} \int_{D} n E \bar{E}_{x x} d x\right) \\
& +\frac{\varepsilon}{2 \lambda^{2}}\|m\|_{0}^{2}+\frac{\varepsilon^{3}}{2 \lambda^{2}}\|n\|_{0}^{2}+\frac{\varepsilon(1-\varepsilon \alpha)}{2}\left\|n_{x}\right\|_{0}^{2} \\
& +\gamma\left\|E_{x x}\right\|_{0}^{2}-2 \gamma \operatorname{Re} \int_{D} n E \bar{E}_{x x} d x \\
& \leq C\|f\|_{0}^{2}+\int_{D} \dot{W}_{1} m d x+\frac{\lambda^{2}}{2}\left\|q_{1}\right\|_{0}^{2}+C\left\|E_{x}\right\|_{0}^{6} \\
& +\left\|q_{2}\right\|_{2}^{2}+C\left(\|n\|_{0}^{12}+\left\|E_{x}\right\|_{0}^{8}\right)+C\left(\|n\|_{0}^{4}+\left\|E_{x}\right\|_{0}^{4}+\left\|g_{x}\right\|_{0}^{4}\right) \\
& -2 \operatorname{Im} \int_{D} n \bar{E}_{x x} \dot{W}_{2} d x+C\left\|q_{2 x x}\right\|_{0}^{4}+2 \operatorname{Im} \int_{D} \dot{W}_{2} \bar{g}_{x x} d x \\
& +C\left(\left\|g_{x x}\right\|_{0}^{2}+\left\|g_{x x}\right\|_{0}^{4}+\|E\|_{0}^{4}+\left\|E_{x}\right\|_{0}^{4}\right) \\
& +2 \operatorname{Re} \int_{D} \dot{W}_{2}\left(\bar{E}_{t x x}+\gamma \bar{E}_{x x}\right) d x \\
& \leq C\left(\|f\|_{0},\left\|q_{1}\right\|_{0},\left\|q_{2}\right\|_{2},\left\|g_{x}\right\|_{0},\left\|g_{x x}\right\|_{0},\right) \\
& +C\left(\left\|E_{x}\right\|_{0},\|n\|_{0},\|E\|_{0}\right)+\int_{D} \dot{W}_{1} m d x \\
& -2 \operatorname{Im} \int_{D} n \bar{E}_{x x} \dot{W}_{2} d x+2 \operatorname{Re} \int_{D} \dot{W}_{2}\left(\bar{E}_{t x x}+\gamma \bar{E}_{x x}\right) d x .
\end{aligned}
$$

Now, taking $\beta=\min \{\varepsilon, \gamma\}$ and letting $H_{1}(t)=\left(1 / 2 \lambda^{2}\right)$ $\|m\|_{0}^{2}+\left(\varepsilon^{2} / 2 \lambda^{2}\right)\|n\|_{0}^{2}+((1-\varepsilon \alpha) / 2)\left\|n_{x}\right\|_{0}^{2}+\left\|E_{x x}\right\|_{0}^{2}-2 \operatorname{Re}$ $\int_{D} n E \bar{E}_{x x} d x$, by (76), the above inequality is changed into

$$
\begin{aligned}
& \frac{d}{d t} H_{1}(t)+\beta H_{1}(t) \\
& \leq C\left(\|f\|_{0},\left\|q_{1}\right\|_{0},\left\|q_{2}\right\|_{2},\left\|g_{x}\right\|_{0},\left\|g_{x x}\right\|_{0}\right) \\
& \quad+C\left(\left\|E_{x}\right\|_{0},\|n\|_{0},\|E\|_{0}\right)+\int_{D} \dot{W}_{1} m d x \\
& \quad-2 \operatorname{Im} \int_{D} n \bar{E}_{x x} \dot{W}_{2} d x+2 \operatorname{Re} \int_{D} \dot{W}_{2}\left(\bar{E}_{t x x}+\gamma \bar{E}_{x x}\right) d x \\
& \leq C\left(f, g, q_{1}, q_{2}, E_{0}, n_{0}, n_{1}\right)+\int_{D} \dot{W}_{1} m d x \\
& -2 \operatorname{Im} \int_{D} n \bar{E}_{x x} \dot{W}_{2} d x+2 \operatorname{Re} \int_{D} \dot{W}_{2}\left(\bar{E}_{t x x}+\gamma \bar{E}_{x x}\right) d x .
\end{aligned}
$$

Integrating (77) from 0 to $t$ and taking expectation on both sides of the above inequality, we get

$$
\begin{gathered}
\mathbb{E} H_{1}(t)-\mathbb{E} H_{1}(0)+\beta \mathbb{E} \int_{0}^{t} H_{1}(s) d s \\
\leq C\left(f, g, q_{1}, q_{2}, E_{0}, n_{0}, n_{1}\right) t .
\end{gathered}
$$


By Gronwall inequality, we have

$$
\begin{aligned}
\mathbb{E} H_{1}(t) & \leq e^{-\beta t} \mathbb{E} H_{1}(0)+C\left(f, g, q_{1}, q_{2}, E_{0}, n_{0}, n_{1}\right) \\
& \leq C\left(f, g, q_{1}, q_{2}, E_{0}, n_{0}, n_{1}\right),
\end{aligned}
$$

where $C\left(f, g, q_{1}, q_{2}, E_{0}, n_{0}, n_{1}\right)$ is independent of $T$.

Since

$$
\begin{aligned}
\left|2 \operatorname{Re} \int_{D} n E \bar{E}_{x x} d x\right| & \leq 2\|E\|_{L_{\infty}}\|n\|_{0}\left\|E_{x x}\right\|_{0} \\
& \leq C\left\|E_{x}\right\|_{0}\|n\|_{0}\left\|E_{x x}\right\|_{0} \\
& \leq \frac{1}{2}\left\|E_{x x}\right\|_{0}^{2}+C\left(\left\|E_{x}\right\|_{0}^{4}+\|n\|_{0}^{4}\right),
\end{aligned}
$$

we have

$$
\begin{aligned}
& C\left(\|m\|_{0}^{2}+\|n\|_{0}^{2}+\left\|n_{x}\right\|_{0}^{2}+\left\|E_{x x}\right\|_{0}^{2}\right)-C\left(\left\|E_{x}\right\|_{0}^{4}+\|n\|_{0}^{4}\right) \\
& \leq H_{1}(t) \leq C\left(\|m\|_{0}^{2}+\|n\|_{0}^{2}+\left\|n_{x}\right\|_{0}^{2}+\left\|E_{x x}\right\|_{0}^{2}\right) \\
& \quad+C\left(\left\|E_{x}\right\|_{0}^{4}+\|n\|_{0}^{4}\right) .
\end{aligned}
$$

By (77) and (81), we get

$$
\begin{aligned}
\frac{d}{d t} H_{1}(t) \leq & C\left(f, g, q_{1}, q_{2}, E_{0}, n_{0}, n_{1}\right) \\
& +\int_{D} \dot{W}_{1} m d x-2 \operatorname{Im} \int_{D} n \bar{E}_{x x} \dot{W}_{2} d x \\
& +2 \operatorname{Re} \int_{D} \dot{W}_{2}\left(\bar{E}_{t x x}+\gamma \bar{E}_{x x}\right) d x
\end{aligned}
$$

By (28), (54), (79), and (81), we get

$$
\begin{aligned}
& \mathbb{E}\left(\|m\|_{0}^{2}+\|n\|_{0}^{2}+\left\|n_{x}\right\|_{0}^{2}+\left\|E_{x x}\right\|_{0}^{2}\right) \\
& \quad \leq \mathbb{E} H_{1}(t)+C \mathbb{E}\left(\left\|E_{x}\right\|_{0}^{4}+\|n\|_{0}^{4}\right) \\
& \quad \leq C\left(f, g, q_{1}, q_{2}, E_{0}, n_{0}, n_{1}\right),
\end{aligned}
$$

where $C\left(f, g, q_{1}, q_{2}, E_{0}, n_{0}, n_{1}\right)$ is independent of $T$.

By (83), we obtain $\left(n_{t}, n, E\right) \in L^{\infty}\left(0, \infty ; L^{2}\left(\Omega, \mathscr{E}_{1}\right)\right)$.

Further, we give an estimate of $H_{1}^{p}(t)$ for any $p \geq 1$. First as

$$
\begin{aligned}
\|n E\|_{0}^{2} & =\int_{D} n E n \bar{E} d x \leq\|n E\|_{0}\|n\|_{0}\|E\|_{L^{\infty}} \\
& \leq C\|n E\|_{0}\|n\|_{0}\left\|E_{x}\right\|_{0^{\prime}} \\
\left\|n E_{x x}\right\|_{-1}^{2} & =\int_{D}(-\Delta)^{-1}\left(n E_{x x}\right)\left(n \bar{E}_{x x}\right) d x \\
& \leq\left\|(-\Delta)^{-1} n E\right\|_{L^{\infty}}\|n\|_{0}\left\|E_{x x}\right\|_{0} \\
& \leq C\left\|(-\Delta)^{-1} n E_{x x}\right\|_{1}\|n\|_{0}\left\|E_{x x}\right\|_{0} \\
& \leq C\left\|n E_{x x}\right\|_{-1}\|n\|_{0}\left\|E_{x x}\right\|_{0} .
\end{aligned}
$$

By (81) and (84), we have

$$
\begin{aligned}
\|n E\|_{0}^{2} & \leq C\|n\|_{0}^{2}\left\|E_{x}\right\|_{0}^{2} \\
& \leq C\left(H_{1}(t)+\left\|E_{x}\right\|_{0}^{4}+\|n\|_{0}^{4}\right)\left\|E_{x}\right\|_{0}^{2},
\end{aligned}
$$

$$
\begin{aligned}
\left\|n E_{x x}\right\|_{-1}^{2} & \leq C\|n\|_{0}^{2}\left\|E_{x x}\right\|_{0}^{2} \\
& \leq C\left(H_{1}(t)+\left\|E_{x}\right\|_{0}^{4}+\|n\|_{0}^{4}\right)\left\|E_{x x}\right\|_{0}^{2},
\end{aligned}
$$

$$
\begin{array}{r}
\|m\|_{0}^{2}+\|n\|_{0}^{2}+\left\|n_{x}\right\|_{0}^{2}+\left\|E_{x x}\right\|_{0}^{2} \\
\leq C\left(H_{1}(t)+\left\|E_{x}\right\|_{0}^{4}+\|n\|_{0}^{4}\right) .
\end{array}
$$

Applying Itô's formula to $H_{1}^{p}(t)$, by (77), (85), (86), (87), and Young inequality, we obtain

$$
\begin{aligned}
& \frac{d}{d t} H_{1}^{p}(t)=p H_{0}^{p-1}(t) \frac{d}{d t} H_{1}(t)+\frac{1}{2} p(p-1) C H_{0}^{p-2}(t) \\
& \cdot\left(\|E\|_{2}^{2}\left\|q_{2}\right\|_{2}^{2}+\|g\|_{1}^{2}\left\|q_{2}\right\|_{1}^{2}+\|n E\|_{0}^{2}\left\|q_{2}\right\|_{2}^{2}\right) \\
& +\frac{1}{2} p(p-1) \mathrm{CH}_{0}^{p-2}(t) \\
& \cdot\left(\left\|n E_{x x}\right\|_{-1}^{2}\left\|q_{2}\right\|_{1}^{2}+\|m\|_{0}^{2}\left\|q_{1}\right\|_{0}^{2}\right) \\
& =p H_{0}^{p-1}(t)\left[-\beta H_{1}(t)+C\left(f, g, q_{1}, q_{2}, E_{0}, n_{0}, n_{1}\right)\right. \\
& \left.+\int_{D} \dot{W}_{1} m d x\right]+p H_{0}^{p-1}(t)\left[-2 \operatorname{Im} \int_{D} n \bar{E}_{x x} \dot{W}_{2} d x\right. \\
& \left.+2 \operatorname{Re} \int_{D} \dot{W}_{2}\left(\bar{E}_{t x x}+\gamma \bar{E}_{x x}\right) d x\right]+\frac{1}{2} p(p-1) C H_{0}^{p-2} \\
& \cdot(t)\left(\|E\|_{2}^{2}\left\|q_{2}\right\|_{2}^{2}+\|g\|_{1}^{2}\left\|q_{2}\right\|_{1}^{2}+\|n E\|_{0}^{2}\left\|q_{2}\right\|_{2}^{2}\right) \\
& +\frac{1}{2} p(p-1) C H_{0}^{p-2}(t) \\
& \cdot\left(\left\|n E_{x x}\right\|_{-1}^{2}\left\|q_{2}\right\|_{1}^{2}+\|m\|_{0}^{2}\left\|q_{1}\right\|_{0}^{2}\right) \\
& \leq-\beta p H_{0}^{p}(t)+C\left(f, g, q_{1}, q_{2}, E_{0}, n_{0}, n_{1}\right) \\
& +p H_{0}^{p-1}(t) \int_{D} \dot{W}_{1} m d x+p H_{0}^{p-1}(t) \\
& \cdot\left[-2 \operatorname{Im} \int_{D} n \bar{E}_{x x} \dot{W}_{2} d \mathrm{x}+2 \operatorname{Re} \int_{D} \dot{W}_{2}\left(\bar{E}_{t x x}\right.\right. \\
& \left.\left.+\gamma \bar{E}_{x x}\right) d x\right]+\frac{1}{2} p(p-1) C H_{0}^{p-2}(t) \\
& \cdot\left(\|E\|_{2}^{2}\left\|q_{2}\right\|_{2}^{2}+\|g\|_{1}^{2}\left\|q_{2}\right\|_{1}^{2}+\|n E\|_{0}^{2}\left\|q_{2}\right\|_{2}^{2}\right) \\
& +\frac{1}{2} p(p-1) C H_{0}^{p-2}(t) \\
& \cdot\left(\left\|n E_{x x}\right\|_{-1}^{2}\left\|q_{2}\right\|_{1}^{2}+\|m\|_{0}^{2}\left\|q_{1}\right\|_{0}^{2}\right) \\
& \leq-\beta p H_{0}^{p}(t)+C\left(f, g, q_{1}, q_{2}, E_{0}, n_{0}, n_{1}\right) \\
& +p H_{0}^{p-1}(t) \int_{D} \dot{W}_{1} m d x+p H_{0}^{p-1}(t)
\end{aligned}
$$




$$
\begin{aligned}
& \cdot\left[-2 \operatorname{Im} \int_{D} n \bar{E}_{x x} \dot{W}_{2} d x+2 \operatorname{Re} \int_{D} \dot{W}_{2}\left(\bar{E}_{t x x}\right.\right. \\
& \left.\left.+\gamma \bar{E}_{x x}\right) d x\right]+\frac{1}{2} p(p-1) C H_{0}^{p-2}(t)\|E\|_{2}^{2}\left\|q_{2}\right\|_{2}^{2} \\
& +\frac{1}{2} p(p-1) C H_{0}^{p-2}(t)\|g\|_{1}^{2}\left\|q_{2}\right\|_{1}^{2} \\
& +\frac{1}{2} p(p-1) C H_{0}^{p-2}(t)\|n E\|_{0}^{2}\left\|q_{2}\right\|_{2}^{2} \\
& +\frac{1}{2} p(p-1) C H_{0}^{p-2}(t)\left\|n E_{x x}\right\|_{-1}^{2}\left\|q_{2}\right\|_{1}^{2} \\
& +\frac{1}{2} p(p-1) C H_{0}^{p-2}(t)\|m\|_{0}^{2}\left\|q_{1}\right\|_{0}^{2} \\
& \leq-\beta p H_{0}^{p}(t)+C\left(f, g, q_{1}, q_{2}, E_{0}, n_{0}, n_{1}\right) \\
& +p H_{0}^{p-1}(t) \int_{D} \dot{W}_{1} m d x+p H_{0}^{p-1}(t) \\
& \cdot\left[-2 \operatorname{Im} \int_{D} n \bar{E}_{x x} \dot{W}_{2} d x+2 \operatorname{Re} \int_{D} \dot{W}_{2}\left(\bar{E}_{t x x}\right.\right. \\
& \left.\left.+\gamma \bar{E}_{x x}\right) d x\right]+\frac{1}{2} p(p-1) C H_{0}^{p-2}(t)\left(H_{1}(t)\right. \\
& \left.+\left\|E_{x}\right\|_{0}^{4}+\|n\|_{0}^{4}\right)\left\|q_{2}\right\|_{2}^{2}+\frac{1}{2} p(p-1) C H_{0}^{p-2} \\
& \cdot(t)\|g\|_{1}^{2}\left\|q_{2}\right\|_{1}^{2}+\frac{1}{2} p(p-1) C H_{0}^{p-2}(t)\left(H_{1}(t)\right. \\
& \left.+\left\|E_{x}\right\|_{0}^{4}+\|n\|_{0}^{4}\right)\left\|E_{x}\right\|_{0}^{2}\left\|q_{2}\right\|_{2}^{2}+\frac{1}{2} p(p-1) \\
& \text { - } C H_{0}^{p-2}(t)\left(H_{1}(t)+\left\|E_{x}\right\|_{0}^{4}+\|n\|_{0}^{4}\right) H_{1}(t)\left\|q_{2}\right\|_{1}^{2} \\
& +\frac{1}{2} p(p-1) C H_{0}^{p-2}(t)\left(H_{1}(t)+\left\|E_{x}\right\|_{0}^{4}\right. \\
& \left.+\|n\|_{0}^{4}\right)\left\|E_{x}\right\|_{0}^{4}\left\|q_{2}\right\|_{1}^{2}+\frac{1}{2} p(p-1) C H_{0}^{p-2}(t) \\
& \cdot\left(H_{1}(t)+\left\|E_{x}\right\|_{0}^{4}+\|n\|_{0}^{4}\right)\|n\|_{0}^{4}\left\|q_{2}\right\|_{1}^{2}+\frac{1}{2} p \\
& \cdot(p-1) C H_{0}^{p-2}(t)\left(H_{1}(t)+\left\|E_{x}\right\|_{0}^{4}+\|n\|_{0}^{4}\right)\left\|q_{1}\right\|_{0}^{2} \\
& \leq-\frac{\beta p}{2} H_{0}^{p}(t)+C\left(f, g, q_{1}, q_{2}, E_{0}, n_{0}, n_{1}\right) \\
& +p H_{0}^{p-1}(t) \int_{D} \dot{W}_{1} m d x+p H_{0}^{p-1}(t) \\
& \cdot\left[-2 \operatorname{Im} \int_{D} n \bar{E}_{x x} \dot{W}_{2} d x+2 \operatorname{Re} \int_{D} \dot{W}_{2}\left(\bar{E}_{t x x}\right.\right. \\
& \left.\left.+\gamma \bar{E}_{x x}\right) d x\right]+C\left(\left\|E_{x}\right\|_{0}^{4 p}+\left\|E_{x}\right\|_{0}^{6 p}+\|n\|_{0}^{4 p}\right) .
\end{aligned}
$$

Taking expectation on both sides of the above inequality, by Hölder inequality and (88), we derive

$$
\begin{aligned}
\mathbb{E} H_{1}^{p}(t) \leq & \mathbb{E} H_{1}^{p}(0)-\frac{\beta p}{2} \mathbb{E} \int_{0}^{t} H_{1}^{p}(s) d s \\
& +C\left(E_{0}, f, g, q_{1}, q_{2}, n_{0}, n_{1}\right) t .
\end{aligned}
$$

By Gronwall inequality, we have

$$
\begin{aligned}
\mathbb{E} H_{1}^{p}(t) & \leq e^{-\beta p t / 2} \mathbb{E} H_{1}^{p}(0)+C\left(E_{0}, f, g, q_{1}, q_{2}, n_{0}, n_{1}\right) \\
& \leq C\left(E_{0}, f, g, q_{1}, q_{2}, n_{0}, n_{1}\right),
\end{aligned}
$$

where $C\left(E_{0}, f, g, q_{1}, q_{2}, n_{0}, n_{1}\right)$ is independent of $T$.

By (87) and (90), we obtain $\left(n_{t}, n, E\right) \in L^{\infty}\left(0, \infty ; L^{2 p}\right.$ $\left.\left(\Omega, \mathscr{C}_{1}\right)\right)$.

By the same approach of Lemma 4, we obtain

$$
\mathbb{E} \sup _{0 \leq t \leq T} H_{1}(t) \leq C_{T}\left(E_{0}, f, g, q_{1}, q_{2}, n_{1}, n_{0}\right)
$$

where $C_{T}$ depends on the initial data. Therefore, by (47), we have

$$
\mathbb{E} \sup _{0 \leq t \leq T}\left\|\left(n_{t}, n, E\right)\right\|_{\mathscr{E}_{1}}^{2} \leq C_{T}\left(E_{0}, f, g, q_{1}, q_{2}, n_{1}, n_{0}\right) .
$$

By (92), we obtain $E \in L^{2}\left(\Omega ; L^{\infty}\left(0, T ; \mathscr{C}_{1}\right)\right)$. Moreover, we get

$$
\begin{aligned}
\mathbb{E} \sup _{0 \leq t \leq T} H_{1}^{p}(t) & \leq C \mathbb{E} H_{1}^{p}(0)+C_{T}\left(E_{0}, f, g, q_{1}, q_{2}, n_{1}, n_{0}\right) \\
& \leq C_{T}\left(E_{0}, f, g, q_{1}, q_{2}, n_{1}, n_{0}\right) .
\end{aligned}
$$

By (87), we have

$$
\begin{aligned}
& C \mathbb{E} \sup _{0 \leq t \leq T}\left(\|m\|_{0}^{2 p}+\|n\|_{0}^{2 p}+\left\|n_{x}\right\|_{-1}^{2 p}+\left\|E_{x x}\right\|_{0}^{2 p}\right) \\
& \quad \leq C \mathbb{E} \sup _{0 \leq t \leq T}\left[C\left(H_{1}(t)+\left\|E_{x}\right\|_{0}^{4}+\|n\|_{0}^{4}\right)\right]^{2 p} \\
& \leq C \mathbb{E} \sup _{0 \leq t \leq T}\left(H_{1}^{2 p}(t)+\left\|E_{x}\right\|_{0}^{8 p}+\|n\|_{0}^{8 p}\right) \\
& \quad \leq C_{T}\left(E_{0}, f, g, q_{1}, q_{2}, n_{1}, n_{0}\right),
\end{aligned}
$$

where $C_{T}$ depends on the initial data. Therefore, by (94), we have

$$
\mathbb{E} \sup _{0 \leq t \leq T}\left\|\left(n_{t}, n, E\right)\right\|_{\mathscr{E}_{1}}^{2 p} \leq C_{T}\left(E_{0}, f, g, q_{1}, q_{2}, n_{1}, n_{0}\right) .
$$

By (95), we obtain $E \in L^{2 p}\left(\Omega ; L^{\infty}\left(0, T ; \mathscr{C}_{1}\right)\right)$. Lemma 5 is proved completely.

Lemma 6. Assume $\left(n_{1}, n_{0}, E_{0}\right) \in \mathscr{E}_{2}, f \in H_{0}^{1}(D), g, q_{1} \in H^{2}$ $(D) \cap H_{0}^{1}(D)$, and $q_{2} \in H^{3}(D) \cap H_{0}^{1}(D)$. Then, for any $T>0$ and $p \geq 1, \quad\left(n_{t}, n, E\right) \in L^{2 p}\left(\Omega ; L^{\infty}\left(0, T ; \mathscr{E}_{2}\right)\right) \cap L^{\infty}(0, \infty$; $\left.L^{2 p}\left(\Omega, \mathscr{E}_{2}\right)\right)$. 
Proof. Applying Itô's formula to $\left\|m_{x}\right\|_{0}^{2}$, by (12), we have

$$
\begin{aligned}
\frac{1}{2 \lambda^{2}} \frac{d}{d t}\left\|m_{x}\right\|_{0}^{2}= & -\int_{D} \frac{1}{\lambda^{2}} m_{t} m_{x x} d x+\frac{\lambda^{2}}{2}\left\|q_{1}\right\|_{1}^{2} \\
= & \int_{D}\left[\frac{\varepsilon}{\lambda^{2}} m+\alpha m_{x x}-\frac{\varepsilon^{2}}{\lambda^{2}} n\right]\left(-m_{x x}\right) d x \\
& +\int_{D}\left[(1-\varepsilon \alpha) n_{x x}+|E|_{x x}^{2}+f+\dot{W}_{1}\right] \\
& \cdot\left(-m_{x x}\right) d x+\frac{\lambda^{2}}{2}\left\|q_{1}\right\|_{1}^{2} \\
= & \frac{\varepsilon}{\lambda^{2}}\left\|m_{x}\right\|_{0}^{2}-\alpha\|m\|_{2}^{2}-\frac{\varepsilon^{2}}{\lambda^{2}} \int_{D} n_{x} m_{x} d x \\
& -(1-\varepsilon \alpha) \int_{D} n_{x x} m_{x x} d x \\
& -\int_{D}|E|_{x x}^{2} m_{x x} d x-\int_{D} f m_{x x} d x \\
& -\int_{D} \dot{W}_{1} m_{x x} d x+\frac{\lambda^{2}}{2}\left\|q_{1}\right\|_{1}^{2} .
\end{aligned}
$$

Since $m=n_{t}+\varepsilon n$, from (96), we obtain

$$
\begin{aligned}
\frac{d}{d t}[ & \left.\frac{1}{2 \lambda^{2}}\left\|m_{x}\right\|_{0}^{2}+\frac{\varepsilon^{2}}{2 \lambda^{2}}\left\|n_{x}\right\|_{0}^{2}+\frac{1-\varepsilon \alpha}{2}\left\|n_{x x}\right\|_{0}^{2}\right] \\
= & \frac{\varepsilon}{\lambda^{2}}\left\|m_{x}\right\|_{0}^{2}-\alpha\|m\|_{2}^{2}-\frac{\varepsilon^{3}}{\lambda^{2}}\left\|n_{x}\right\|_{0}^{2} \\
& -\varepsilon(1-\varepsilon \alpha)\left\|n_{x x}\right\|_{0}^{2}-\int_{D}|E|_{x x}^{2} m_{x x} d x \\
& +\int_{D} f_{x} m_{x} d x-\int_{D} \dot{W}_{1} m_{x x} d x+\frac{\lambda^{2}}{2}\left\|q_{1}\right\|_{1}^{2},
\end{aligned}
$$

where $\lambda_{1}$ is the first eigenvalue of $-\Delta$.

By (97), we obtain

$$
\begin{aligned}
& \frac{d}{d t}\left[\frac{1}{2 \lambda^{2}}\left\|m_{x}\right\|_{0}^{2}+\frac{\varepsilon^{2}}{2 \lambda^{2}}\left\|n_{x}\right\|_{0}^{2}+\frac{1-\varepsilon \alpha}{2}\left\|n_{x x}\right\|_{0}^{2}\right] \\
& \quad-\left(\frac{\varepsilon}{\lambda^{2}}-\alpha \lambda_{1}\right)\left\|m_{x}\right\|_{0}^{2}+\frac{\varepsilon^{3}}{\lambda^{2}}\left\|n_{x}\right\|_{0}^{2}+\varepsilon(1-\varepsilon \alpha)\left\|n_{x x}\right\|_{0}^{2} \\
& \leq-\int_{D}|E|_{x x}^{2} m_{x x} d x+\int_{D} f_{x} m_{x} d x-\int_{D} \dot{W}_{1} m_{x x} d x \\
& \quad+\frac{\lambda^{2}}{2}\left\|q_{1}\right\|_{1}^{2}
\end{aligned}
$$

Applying Itô's formula to $\left\|E_{x x x}\right\|_{0}^{2}$, by (13), we have

$$
\begin{aligned}
\frac{d}{d t}\left\|E_{x x x}\right\|_{0}^{2} & =2 \operatorname{Re} \int_{D} E_{x x x} \bar{E}_{t x x x} d x+\left\|q_{2}\right\|_{3}^{2} \\
& =-2 \operatorname{Re} \int_{D} E_{x x} \bar{E}_{t x x x x} d x+\left\|q_{2}\right\|_{3}^{2} .
\end{aligned}
$$

Substituting $E_{x x}=-i E_{t}+n E-i \gamma E+g+\dot{W}_{2}$ into (99), we have

$$
\begin{aligned}
\frac{d}{d t}\left\|E_{x x x}\right\|_{0}^{2}= & -2 \operatorname{Re} \int_{D}\left(-i E_{t}+n E-i \gamma E+g+\dot{W}_{2}\right) \bar{E}_{t x x x x} d x \\
& +\left\|q_{2}\right\|_{3}^{2}=-2 \operatorname{Re} \int_{D} n E \bar{E}_{t x x x x} d x \\
& -2 \operatorname{Re} \int_{D}\left(\gamma \bar{E}_{x x x x}\right)\left(E_{x x}+n E-i \gamma E+g+\dot{W}_{2}\right) d x \\
& -2 \operatorname{Re} \int_{D} g \bar{E}_{t x x x x} d x-2 \operatorname{Re} \int_{D} \dot{W}_{2} \bar{E}_{t x x x x} d x+\left\|q_{2}\right\|_{3}^{2} \\
= & -2 \gamma\left\|E_{x x x}\right\|_{0}^{2}-2 \operatorname{Re} \int_{D} n E\left(\bar{E}_{t x x x x}+\gamma \bar{E}_{x x x x}\right) d x \\
& -2 \operatorname{Re} \int_{D} \dot{W}_{2}\left(\bar{E}_{t x x x x}+\gamma \bar{E}_{x x x x}\right) d x \\
& -2 \operatorname{Re} \int_{D} g\left(\bar{E}_{t x x x x}+\gamma \bar{E}_{x x x x}\right) d x+\left\|q_{2}\right\|_{3}^{2} .
\end{aligned}
$$

From (98) and (100), we obtain

$$
\begin{aligned}
& \frac{d}{d t}\left(\frac{1}{2 \lambda^{2}}\left\|m_{x}\right\|_{0}^{2}+\frac{\varepsilon^{2}}{2 \lambda^{2}}\left\|n_{x}\right\|_{0}^{2}+\frac{1-\varepsilon \alpha}{2}\left\|n_{x x}\right\|_{0}^{2}+\left\|E_{x x x}\right\|_{0}^{2}\right) \\
& \quad-\left(\frac{\varepsilon}{\lambda^{2}}-\alpha \lambda_{1}\right)\left\|m_{x}\right\|_{0}^{2}+\frac{\varepsilon^{3}}{\lambda^{2}}\left\|n_{x}\right\|_{0}^{2}+\varepsilon(1-\varepsilon \alpha)\left\|n_{x x}\right\|_{0}^{2} \\
& \leq-\int_{D}|E|_{x x}^{2} m_{x x} d x+\int_{D} f_{x} m_{x} d x-\int_{D} \dot{W}_{1} m_{x x} d x \\
& \quad+\frac{\lambda^{2}}{2}\left\|q_{1}\right\|_{1}^{2}-2 \gamma\left\|E_{x x x}\right\|_{0}^{2}-2 \operatorname{Re} \int_{D} n E\left(\bar{E}_{t x x x x}+\gamma \bar{E}_{x x x x}\right) d x \\
& \quad-2 \operatorname{Re} \int_{D} \dot{W}_{2}\left(\bar{E}_{t x x x x}+\gamma \bar{E}_{x x x x}\right) d x \\
& \quad-2 \operatorname{Re} \int_{D} g\left(\bar{E}_{t x x x x}+\gamma \bar{E}_{x x x x}\right) d x+\left\|q_{2}\right\|_{3}^{2} .
\end{aligned}
$$

Taking $0<\varepsilon \leq \min \left\{1 / \alpha, \alpha \lambda_{1} \lambda^{2} / 2\right\}$, and by (101), we have

$$
\begin{aligned}
& \frac{d}{d t}\left(\frac{1}{2 \lambda^{2}}\left\|m_{x}\right\|_{0}^{2}+\frac{\varepsilon^{2}}{2 \lambda^{2}}\left\|n_{x}\right\|_{0}^{2}+\frac{1-\varepsilon \alpha}{2}\left\|n_{x x}\right\|_{0}^{2}+\left\|E_{x x x}\right\|_{0}^{2}\right) \\
& \quad+\frac{\varepsilon}{\lambda^{2}}\left\|m_{x}\right\|_{0}^{2}+\frac{\varepsilon^{3}}{\lambda^{2}}\left\|n_{x}\right\|_{0}^{2}+\varepsilon(1-\varepsilon \alpha)\left\|n_{x x}\right\|_{0}^{2}+2 \gamma\left\|E_{x x x}\right\|_{0}^{2} \\
& \leq-\int_{D}|E|_{x x}^{2} m_{x x} d x+\int_{D} f_{x} m_{x} d x-\int_{D} \dot{W}_{1} m_{x x} d x \\
& \quad+\frac{\lambda^{2}}{2}\left\|q_{1}\right\|_{1}^{2}-2 \operatorname{Re} \int_{D} n E\left(\bar{E}_{t x x x x}+\gamma \bar{E}_{x x x x}\right) d x \\
& \quad-2 \operatorname{Re} \int_{D} \dot{W}_{2}\left(\bar{E}_{t x x x x}+\gamma \bar{E}_{x x x x}\right) d x \\
& \quad-2 \operatorname{Re} \int_{D} g\left(\bar{E}_{t x x x x}+\gamma \bar{E}_{x x x x}\right) d x+\left\|q_{2}\right\|_{3}^{2} .
\end{aligned}
$$


Since

$$
\begin{aligned}
& 2 \operatorname{Re} \int_{D} n E\left(\bar{E}_{t x x x x}+\gamma \bar{E}_{x x x x}\right) d x \\
& =2 \operatorname{Re} \int_{D} n_{x x} \mathrm{E}\left(\bar{E}_{t x x}+\gamma \bar{E}_{x x}\right) d x \\
& +2 \operatorname{Re} \int_{D} n E_{x x}\left(\bar{E}_{t x x}+\gamma \bar{E}_{x x}\right) d x \\
& \quad+4 \operatorname{Re} \int_{D} n_{x} E_{x}\left(\bar{E}_{t x x}+\gamma \bar{E}_{x x}\right) d x \\
& \frac{d}{d t} \int_{D} n_{x x}|E|_{x x}^{2} d x \\
& =\int_{D} n_{t x x}|E|_{x x}^{2} d x+2 \operatorname{Re} \int_{D} n_{x x} \bar{E}_{t} E_{x x} d x \\
& \quad+2 \operatorname{Re} \int_{D} n_{x x} \bar{E} E_{t x x} d x-4 \frac{d}{d t} \operatorname{Re} \int_{D} n_{x} E_{x} \bar{E}_{x x} d x \\
& \quad+4 \operatorname{Re} \int_{D} n_{t x} E_{x} \bar{E}_{x x} d x+4 \operatorname{Re} \int_{D} n_{x} q_{2 x} \bar{q}_{2 x x} d x \\
& \quad+2 \operatorname{Re} \int_{D} n_{x x} \bar{q}_{2} q_{2 x x} d x+2 \operatorname{Re} \int_{D} n_{x x} \bar{q}_{2 x} q_{2 x} d x \\
& \frac{d}{d t} \int_{D} n\left|E_{x x}\right|^{2} d x=\int_{D} n_{t}\left|E_{x x}\right|^{2} d x+2 \operatorname{Re} \int_{D} n E_{x x} \bar{E}_{t x x} d x \\
& \quad+\int_{D} n\left|q_{2 x x}\right|^{2} d x, \\
& \frac{d}{d t}\left(2 \operatorname{Re} \int_{D} n_{x} E_{x} \bar{E}_{x x} d x\right)
\end{aligned}
$$

then we deduce

$$
\begin{aligned}
2 \operatorname{Re} \int_{D} n E\left(\bar{E}_{t x x x x}+\gamma \bar{E}_{x x x x}\right) d x \\
=\frac{d}{d t} \int_{D} n_{x x}|E|_{x x}^{2} d x+\frac{d}{d t} \int_{D} n\left|E_{x x}\right|^{2} d x \\
\quad+\frac{d}{d t}\left(8 \operatorname{Re} \int_{D} n_{x} E_{x} \bar{E}_{x x} d x\right)-\int_{D} n_{t x x}|E|_{x x}^{2} d x \\
\quad-2 \operatorname{Re} \int_{D} n_{x x} \bar{E}_{t} E_{x x} d x-4 \operatorname{Re} \int_{D} n_{t x} E_{x} \bar{E}_{x x} d x \\
\quad-\int_{D} n_{t}\left|E_{x x}\right|^{2} d x+2 \gamma \operatorname{Re} \int_{D} n_{x x} E \bar{E}_{x x} d x \\
\quad-4 \operatorname{Re} \int_{D} n_{t x} E_{x} \bar{E}_{x x} d x-4 \operatorname{Re} \int_{D} n_{x} E_{t x} \bar{E}_{x x} d x \\
\quad+2 \gamma \operatorname{Re} \int_{D} n E_{x x} \bar{E}_{x x} d x+4 \gamma \operatorname{Re} \int_{D} n_{x} E_{x} \bar{E}_{x x} d x \\
\quad-4 \operatorname{Re} \int_{D} n_{x} q_{2 x} \bar{q}_{2 x x} d x-\int_{D} n\left|q_{2 x x}\right|^{2} d x \\
\quad-4 \operatorname{Re} \int_{D} n_{x} q_{2 x} \bar{q}_{2 x x} d x-2 \operatorname{Re} \int_{D} n_{x x} \bar{q}_{2} q_{2 x x} d x \\
\quad-2 \operatorname{Re} \int_{D} n_{x x} \bar{q}_{2 x} q_{2 x} d x .
\end{aligned}
$$

Substituting (104) into (102), we have

$$
\begin{aligned}
\frac{d}{d t}\left(\frac{1}{2 \lambda^{2}}\left\|m_{x}\right\|_{0}^{2}+\frac{\varepsilon^{2}}{2 \lambda^{2}}\left\|n_{x}\right\|_{0}^{2}+\frac{1-\varepsilon \alpha}{2}\left\|n_{x x}\right\|_{0}^{2}+\left\|E_{x x x}\right\|_{0}^{2}\right) \\
\cdot \frac{d}{d t}\left(\int_{D} n_{x x}|E|_{x x}^{2} d x+\int_{D} n\left|E_{x x}\right|^{2} d x+8 \operatorname{Re} \int_{D} n_{x} E_{x} \bar{E}_{x x} d x\right) \\
\quad+\frac{\varepsilon}{\lambda^{2}}\left\|m_{x}\right\|_{0}^{2}+\frac{\varepsilon^{3}}{\lambda^{2}}\left\|n_{x}\right\|_{0}^{2}+\varepsilon(1-\varepsilon \alpha)\left\|n_{x x}\right\|_{0}^{2}+2 \gamma\left\|E_{x x x}\right\|_{0}^{2} \\
\leq-\int_{D}|E|_{x x}^{2} m_{x x} d x+\int_{D} f_{x} m_{x} d x-\int_{D} \dot{W}_{1} m_{x x} d x \\
\quad+\frac{\lambda^{2}}{2}\left\|q_{1}\right\|_{1}^{2}+\int_{D} n_{t x x}|E|_{x x}^{2} d x+2 \operatorname{Re} \int_{D} n_{x x} \bar{E}_{t} E_{x x} d x \\
\quad+4 \operatorname{Re} \int_{D} n_{t x} E_{x} \bar{E}_{x x} d x+\int_{D} n_{t}\left|E_{x x}\right|^{2} d x \\
\quad-2 \gamma \operatorname{Re} \int_{D} n_{x x} E \bar{E}_{x x} d x+4 \operatorname{Re} \int_{D} n_{t x} E_{x} \bar{E}_{x x} d x \\
\quad+4 \operatorname{Re} \int_{D} n_{x} E_{t x} \bar{E}_{x x} d x-2 \gamma \operatorname{Re} \int_{D} n E_{x x} \bar{E}_{x x} d x \\
\quad-4 \gamma \operatorname{Re} \int_{D} n_{x} E_{x} \bar{E}_{x x} d x+4 \operatorname{Re} \int_{D} n_{x} q_{2 x} \bar{q}_{2 x x} d x \\
\quad+\int_{D} n\left|q_{2 x x}\right|^{2} \mathrm{~d} x+4 \operatorname{Re} \int_{D} n_{x} q_{2 x} \bar{q}_{2 x x} d x \\
\quad+2 \operatorname{Re} \int_{D} n_{x x} \bar{q}_{2} q_{2 x x} d x+2 \operatorname{Re} \int_{D} n_{x x} \bar{q}_{2 x} q_{2 x} d x \\
\quad-2 \operatorname{Re} \int_{D} \dot{W}_{2}\left(\bar{E}_{t x x x x}+\gamma \bar{E}_{x x x x}\right) d x \\
\quad-2 \operatorname{Re} \int_{D} g\left(\bar{E}_{t x x x x}+\gamma \bar{E}_{x x x x}\right) d x+\left\|q_{2}\right\|_{3}^{2} . \\
\quad
\end{aligned}
$$

\section{Existence and Uniqueness of Solutions}

By the above a priori estimates, we prove the existence and uniqueness of solutions for stochastic Equations (7) and (8) with initial boundary conditions (9) and (10) in spaces $\mathscr{E}_{1}$.

Theorem 7. Assume $\left(n_{1}, n_{0}, E_{0}\right) \in \mathscr{E}_{1}, f \in L^{2}(D), q_{1} \in H_{0}^{1}(D)$, and $g, q_{2} \in H^{2}(D) \cap H_{0}^{1}(D)$. Then, there is a unique solution $\left(n_{t}, n, E\right) \in L^{\infty}\left(\mathbb{R}^{+}, \mathscr{C}_{1}\right)$ almost surely for Equations (7) and (8). And $\left(n_{t}, n, E\right)$ is continuous from $\mathbb{R}^{+}$to $\mathscr{E}_{1}$.

Proof. First, we assume $\left(n_{1}, n_{0}, E_{0}\right) \in \mathscr{E}_{1}$. Let $\left\{e_{i}\right\}_{i=1}^{\infty}$ be the eigenvectors of Laplace operator $-\Delta$ on $D$ with Dirichlet boundary condition, which is also an orthonormal basis of $L^{2}(D)$. Let $P^{k}$ be the projection from $L^{2}(D)$ onto the space spanned by $\left\{e_{i}: i=1,2, \cdots, k\right\}$. We define $\left(n_{t}^{k}, n^{k}, E^{k}\right)$ as the Galerkin approximate solution of the following equations:

$\frac{1}{\lambda^{2}} n_{t t}^{k}-n_{x x}^{k}-P^{k}\left|E^{k}\right|_{x x}^{2}-\alpha n_{t x x}^{k}=f^{k}+\dot{W}_{1}^{k}$, 


$$
\begin{aligned}
i E_{t}^{k} & +E_{x x}^{k}-P^{k}\left(n^{k} E^{k}\right)+i \gamma E^{k} \\
& =g^{k}+\dot{W}_{2}^{k}, \quad t \in R^{+}, x \in D, \\
n^{k}(0) & =P^{k} n_{0}, n_{t}^{k}(0)=P^{k} n_{1}, E^{k}(0)=\mathrm{P}^{k} E_{0} .
\end{aligned}
$$

Here, $f^{k}=P^{k} f, g^{k}=P^{k} g, \dot{W}_{1}^{k}=P^{k} \dot{W}_{1}$, and $\dot{W}_{2}^{k}=P^{k} \dot{W}_{2}$, and $P^{k}$ commutes with the operator $\Delta$. Now, we will treat the above equations pathwise by introducing the random processes solving

$$
\begin{aligned}
\frac{1}{\lambda^{2}} \eta_{t t}-\eta_{x x}-\alpha \eta_{t x x} & =\dot{W}_{1}, \\
i \xi_{t}+\xi_{x x}+i \gamma \xi & =\dot{W}_{2}
\end{aligned}
$$

with Dirichlet boundary conditions

$$
\left.\eta\right|_{\partial D}=0,\left.\xi\right|_{\partial D}=0
$$

and initial conditions

$$
\eta(x, 0)=0, \eta_{t}(x, 0)=0, \xi(x, 0)=0, \quad x \in D .
$$

Following the same method as in Section 3, for any $T>0$ and almost all sample point $\omega \in \Omega$, we have

$$
\begin{aligned}
& \eta_{t} \in C\left(0, T ; H^{1}(D)\right), \eta \in C\left(0, T ; H^{1}(D) \cap H_{0}^{1}(D)\right) \\
& \xi \in C\left(0, T ; H^{3}(D) \cap H_{0}^{1}(D)\right)
\end{aligned}
$$

and satisfy the following estimates:

$$
\mathbb{E}\left(\left\|\eta_{t}(t)\right\|_{1}^{2}+\|\eta(t)\|_{2}^{2}+\|\xi(t)\|_{3}^{2}\right) \leq C
$$

for some positive constant $C$ independent of $T$. And for any $T>0$,

$$
\mathbb{E} \sup _{0 \leq t \leq T}\left(\left\|\eta_{t}(t)\right\|_{1}^{2}+\|\eta(t)\|_{2}^{2}+\|\xi(t)\|_{3}^{2}\right) \leq C_{T}
$$

for some positive constant $C_{T}$. Now, let $R^{k, M}=\left(N_{t}^{k, M}, N^{k, M}\right.$, $\left.F^{k, M}\right)$ be the solution of the following equations:

$$
\begin{gathered}
\frac{1}{\lambda^{2}} N_{t t}^{k, M}-N_{x x}^{k, M}-\chi_{M}\left(\left|R^{k, M}\right|_{\mathscr{C}_{1}}\right) P^{k}\left|E^{k}\right|_{x x}^{2}-\alpha N_{t x x}^{k, M}=f^{k}, \\
i F_{t}^{k, M}+F_{x x}^{k, M}-\chi_{M}\left(\left|R^{k, M}\right|_{\mathscr{C}_{1}}\right) P^{k}\left(n^{k, M} E^{k, M}\right)+i \gamma F^{k, M}=g^{k},
\end{gathered}
$$

with initial conditions

$$
N_{t}^{k, M}(x, 0)=P^{k} n_{1}, N^{k, M}(x, 0)=P^{k} n_{0}, F^{k, M}(x, 0)=P^{k} E_{0} .
$$

Here, $\quad n^{k, M}=N^{k, M}+P^{k} \eta$ and $E^{k, M}=F^{k, M}+P^{k} \xi$. And $\chi_{M} \in C_{0}^{\infty}(\mathbb{R})$ such that $\chi_{M}=1$ for $|r| \leq M$ and $\chi_{M}=0$ for $|r| \geq M$. Note that (116) and (117) are random differential equations with Lipschitz nonlinearity in finite dimension. Then, for almost all sample point $\omega \in \Omega$, we have a unique solution $\left(N_{t}^{k, M}, N^{k, M}, F^{k, M}\right)$ for (116) and (117). Define the stopping time by $\tau_{M}=\inf \left\{t>0:|R|_{\mathscr{C}_{1}}^{k, M} \geq M\right\}$, if the set $\left\{|R|_{\mathscr{E}_{1}}^{k, M} \geq M\right\}$ is nonempty; otherwise, $\tau_{M}=\infty$.

Since $\tau_{M}$ is increasing in $M$, let $\tau_{\infty}=\lim _{M \rightarrow \infty} \tau_{M}$ almost surely. And for $t<\tau_{M}$, we have

$$
\left(N_{t}^{k, M}, N^{k, M}, F^{k, M}\right)+\left(P^{k} \eta_{t}, P^{k} \eta, P^{k} \xi\right)
$$

satisfying (106) and (107). By the estimates given in Section 3 and (114) and (115), for any $t \geq 0$, we have

$$
\mathbb{E}\left|\left(N_{t}^{k, M}, N^{k, M}, F^{k, M}\right)\right|_{\mathscr{E}_{1}}^{2} \leq C\left(n_{0}, n_{1}, E_{0}, f, g, q_{1}, q_{2}\right)
$$

with $C\left(n_{0}, n_{1}, E_{0}, f, g, q_{1}, q_{2}\right)$ independent of $T$ and $M$, and for any $T>0$,

$\mathbb{E} \sup _{0 \leq t \leq T \wedge \tau_{M}}\left|\left(N_{t}^{k, M}, N^{k, M}, F^{k, M}\right)\right|_{\mathscr{E}_{1}}^{2} \leq C_{T}\left(n_{0}, n_{1}, E_{0}, f, g, q_{1}, q_{2}\right)$,

with $C\left(n_{0}, n_{1}, E_{0}, f, g, q_{1}, q_{2}\right)$ independent of $M$; here, $T \wedge$ $\tau_{M}=\min \left\{T, \tau_{M}\right\}$. On the other hand, we have

$$
\begin{gathered}
\mathbb{E}\left|\left(N_{t}^{k, M}\left(T \wedge \tau_{M}\right), N^{k, M}\left(T \wedge \tau_{M}\right), F^{k, M}\left(T \wedge \tau_{M}\right)\right)\right|_{\mathscr{E}_{1}}^{2} \\
\geq \mathbb{E}\left[I\left(\tau_{M} \leq T\right) \mid\left(N_{t}^{k, M}\left(T \wedge \tau_{M}\right), N^{k, M}\left(T \wedge \tau_{M}\right),\right.\right. \\
\left.\left.F^{k, M}\left(T \wedge \tau_{M}\right)\right)\left.\right|_{\mathscr{E}_{1}} ^{2}\right] \geq M^{2} \mathbb{P}\left(\tau_{M} \leq T\right),
\end{gathered}
$$

where $I\left(\tau_{M} \leq T\right)=1$ for $\tau_{M} \leq T$ and $I\left(\tau_{M} \leq T\right)=0$ for $\tau_{M}>$ $T$. Then, according to (120), we have

$$
\mathbb{P}\left(\tau_{M} \leq T\right) \leq \frac{1}{M^{2}} C\left(n_{0}, n_{1}, E_{0}, f, g, q_{1}, q_{2}\right)
$$

According to the above estimate and Borel-Cantelli lemma, for any $T>0$, we have $\mathbb{P}\left(\tau_{M}>T\right)=1$. So we know

$$
\left(N_{t}^{k}, N^{k}, F^{k}\right)=\lim _{M \rightarrow \infty}\left(N_{t}^{k, M}, N^{k, M}, F^{k, M}\right)
$$

satisfying the following random differential equations:

$$
\begin{gathered}
\frac{1}{\lambda^{2}} N_{t t}^{k}-N_{x x}^{k}-P^{k}\left|E^{k}\right|_{x x}^{2}-\alpha N_{t x x}^{k}=f^{k}, \\
i F_{t}^{k}+F_{x x}^{k}-P^{k}\left(n^{k} E^{k}\right)+i \gamma F^{k}=g^{k},
\end{gathered}
$$


with initial conditions

$$
N^{k}(0)=P^{k} n_{0}, N_{t}^{k}(0)=P^{k} n_{1}, F^{k}(0)=P^{k} E_{0} .
$$

Then, $\left(N_{t}^{k}, N^{k}, F^{k}\right)$ satisfies the estimates (120) and (121), and for any $t \geq 0$,

$$
\begin{aligned}
\left(n_{t}^{k}(t), n^{k}(t), E^{k}(t)\right)= & \left(N_{t}^{k}(t), N^{k}(t), F^{k}(t)\right) \\
& +\left(P^{k} \eta_{t}(t), P^{k} \eta(t), P^{k} \xi^{k}(t)\right),
\end{aligned}
$$

is the unique global solution of (106), (107), and (108). Now, we will consider (125) and (126) for fixed $\omega$. First, by (121), for any $T>0$,

$$
\mathbb{P}\left(\bigcap_{L=1}^{\infty} \bigcup_{l=1}^{\infty} \bigcap_{k=l}^{\infty}\left\{\sup _{0 \leq t \leq T}\left\|\left(N_{t}^{k}(t), N^{k}(t), F^{k}(t)\right)\right\|_{\mathscr{E}_{1}}^{2} \geq L\right\}\right)=0
$$

$$
\begin{aligned}
& \text { Let } \\
& \tilde{\Omega}=\bigcap_{L=1}^{\infty} \bigcup_{l=1}^{\infty} \bigcap_{k=l}^{\infty}\left\{\sup _{0 \leq t \leq T}\left\|\left(N_{t}^{k}(t), N^{k}(t), F^{k}(t)\right)\right\|_{\mathscr{E}_{1}}^{2} \leq L\right\} .
\end{aligned}
$$

Then, $\mathbb{P}(\Omega \tilde{\Omega})=0$. Now, for any fixed $\omega \in \tilde{\Omega}$, there is an $r(\omega)$ with $0<r(\omega)<\infty$ such that

$$
\sup _{0 \leq t \leq T}\left\|\left(N_{t}^{k}(t), N^{k}(t), F^{k}(t)\right)\right\|_{\mathscr{E}_{1}}^{2} \leq r(\omega)
$$

Then, we can extract a subsequence still denoted by $\left(N_{t}^{k}, N^{k}, F^{k}\right)$ such that for any $T>0, N_{t}^{k}$ converges to $N_{t}$ weakly star in $L^{\infty}\left(0, T ; L^{2}(D)\right), N^{k}$ converges to $N$ weakly star in $L^{\infty}\left(0, T ; H_{0}^{1}(D)\right)$, and $F^{k}$ converges to $F$ weakly star in $L^{\infty}\left(0, T ; H^{2} \cap H_{0}^{1}(D)\right)$.

These convergences are sufficient to pass the limit $k \rightarrow$ $\infty$ in linear terms, but we need a strong convergence of $F^{k}$ for nonlinear terms. In fact from (126) and estimate (131), we know $F^{k}$ is bounded in $L^{\infty}\left(0, T ; L^{2}(D)\right)$. Then, by Lemma 1 , we can further extract a subsequence still denoted by $F^{k}$ such that $F^{k}$ converges to $F$ strongly in $L^{2}\left(0, T ; H_{0}^{1}\right.$ $(D))$. Then, by a standard procedure, we can pass the limit $k \rightarrow \infty$ to show that $\left(N_{t}, N, F\right) \in L^{\infty}\left(0, T ; \mathscr{C}_{1}\right)$ is a weak solution of

$$
\begin{array}{r}
\frac{1}{\lambda^{2}} N_{t t}-N_{x x}-|E|_{x x}^{2}-\alpha N_{t x x}=f, \\
i F_{t}+F_{x x}-n E+i \gamma F=g,
\end{array}
$$

with initial conditions

$$
N_{t}(x, 0)=n_{1}(x), N(x, 0)=n_{0}(x), F(x, 0)=E_{0}(x), \quad x \in D .
$$

Then, $\left(n_{t}, n, F\right)=\left(N_{t}, N, F\right)+\left(\eta_{t}, \eta, \xi\right)$ is a solution of (7) and (8) and satisfies the estimates in Section 3.

Now, we prove the continuity of the solution. In fact, for $\omega \in \tilde{\Omega}, f \in L^{2}(D)$, and $|E|_{x x}^{2} \in L^{\infty}\left(0, T ; L_{0}^{2}(D)\right)$, we have

$$
\frac{1}{\lambda^{2}} N_{t t}-N_{x x} \in L^{\infty}\left(0, T ; L_{0}^{2}(D)\right)
$$

Then, by Lemma 4.1 and Chapter II of [23], we have for almost all $\omega \in \Omega$

$$
N \in C\left(0, T ; H_{0}^{1}(D)\right), N_{t} \in C\left(0, T ; L^{2}(D)\right) .
$$

By a similar method, noticing $g \in H_{0}^{1}(D)$ and $F_{t} \in L^{\infty}$ $\left(0, T ; L_{0}^{2}(D)\right)$ almost surely, by $[21]$, we have

$$
F \in C\left(0, T ; H_{0}^{2}(D) \bigcap H_{0}^{1}(D)\right)
$$

Then, by (113) and the definition of $N$ and $F$,

$$
\left(n_{t}, n, F\right) \in C\left(0, T ; \mathscr{E}_{1}\right)
$$

almost surely. Now, as the noise is additive, we can follow the same approach as in [11]. The solution $\left(n_{t}, n, E\right)$ is unique in $L^{\infty}\left(0, T ; \mathscr{E}_{1}\right)$ almost surely and is continuous from $[0, T]$ to $\mathscr{E}_{1}$ almost surely. Then, for $T$ is arbitrary, by the estimates in Section 3, we derive Theorem 7 .

Using the same method as above, we obtain the following results.

Theorem 8. Assume $\left(n_{1}, n_{0}, E_{0}\right) \in \mathscr{E}_{2}, f \in H_{0}^{1}(D), g, q_{1} \in H^{2}$ $(D) \cap H_{0}^{1}(D)$, and $q_{2} \in H^{3}(D) \cap H_{0}^{1}(D)$. Then, there is a unique solution $\left(n_{t}, n, E\right) \in L^{\infty}\left(\mathbb{R}^{+}, \mathscr{E}_{1}\right)$ almost surely for Equations (7) and (8). And $\left(n_{t}, n, E\right)$ is continuous from $\mathbb{R}^{+}$ to $\mathscr{E}_{1}$.

\section{Conclusion}

In [10], the authors studied the initial boundary value problem for a generalized Zakharov system. The authors proved the global existence and uniqueness of the generalized solution to the problem by a priori estimates and Galerkin method. In this paper, we discuss the random forced strongly dissipative Zakharov equations, which are the generalized Zakharov system under random influences in [10]. We proved the existence and uniqueness of solutions in energy spaces $\mathscr{E}_{1}$ and $\mathscr{E}_{2}$. The results of this paper are a good supplement to the results in [10]. 


\section{Conflicts of Interest}

The authors declare that there are no conflicts of interest regarding the publication of this paper.

\section{Acknowledgments}

The authors are grateful to the reviewers for useful remarks and suggestions that greatly improved the presentation of this manuscript. This work is supported by the National Natural Science Foundation of China (no. 11401223), the Natural Science Foundation of Guangdong (no. 2015A030313424), and the Science and Technology Program of Guangzhou (no. 201607010005).

\section{References}

[1] V. E. Zakharov, "Collapse of Langmuir waves," Soviet Journal of Experimental and Theoretical Physics, vol. 35, no. 5, pp. 908-914, 1972.

[2] S. Ma and Q. Chang, "Strange attractors on pseudospectral solutions for dissipative Zakharov equations," Acta Mathematica Scientia, vol. 24, no. 3, pp. 321-336, 2004.

[3] H. Pecher, "An improved local well-posedness result for the one-dimensional Zakharov system," Journal of Mathematical Analysis and Applications, vol. 342, no. 2, pp. 1440-1454, 2008.

[4] B. Guo, J. Zhang, and X. Pu, "On the existence and uniqueness of smooth solution for a generalized Zakharov equation," Journal of Mathematical Analysis and Applications, vol. 365, no. 1, pp. 238-253, 2010.

[5] F. Linares and C. Matheus, "Well-posedness for the 1D Zakharov-Rubenchik system," Advances in Differential Equations, vol. 14, pp. 261-288, 2009.

[6] F. Linares and J. C. Saut, "The Cauchy problem for the 3D Zakharov-Kuznetsov equation," Discrete \& Continuous Dynamical Systems - A, vol. 24, no. 2, pp. 547-565, 2009.

[7] B. L. Guo, "On some problems for a wide class of the system of Zakharov equation," in Proceedings of DD-3, pp. 395-415, Changchun, China, 1982.

[8] B. L. Guo and L. J. Shen, "The existence and uniqueness of the classicial solution on the periodic initial problem for Zakharov equation," Acta Mathematicae Applicatae Sinica, vol. 5, pp. 310-324, 1982.

[9] X. Zheng, Y. Shang, and H. Di, "The time-periodic solutions to the modified Zakharov equations with a quantum correction," Mediterranean Journal of Mathematics, vol. 14, no. 4, p. 152, 2017.

[10] X. Wang and Y. Shang, "On the global existence and small dissipation limit for generalized dissipative Zakharov system," Mathematical Methods in the Applied Sciences, vol. 41, no. 10, pp. 3718-3749, 2018.

[11] I. Flahaut, "Attractors for the dissipative Zakharov system," Nonlinear Analysis: Theory, Methods \& Applications, vol. 16, no. 7-8, pp. 599-633, 1991.

[12] I. D. Chueshov and A. S. Shcherbina, "On 2D Zakharov system in a bounded domain," Differential and Integral Equations, vol. 18, no. 7, pp. 781-812, 2005.

[13] O. Goubet and I. Moise, "Attractor for dissipative Zakharov system," Nonlinear Analysis: Theory, Methods \& Applications, vol. 31, no. 7, pp. 823-847, 1998.
[14] Y. S. Li and B. L. Guo, "Attractor for dissipative Zakharov equations in an unbounded domain," Reviews in Mathematical Physics, vol. 9, no. 6, pp. 675-687, 1997.

[15] L. Arnold, Random Dynamical Systems, Springer-Verlag, 1998.

[16] G. Chen, J. Duan, and J. Zhang, "Geometric shape of invariant manifolds for a class of stochastic partial differential equations," Journal of Mathematical Physics, vol. 52, no. 7, article 072702, 2011.

[17] E. Weinan, X. Li, and E. Vanden-Eijnden, "Some recent progress in multiscale modeling," in Multiscale Modelling and Simulation, vol. 39 of Lecture Notes in Computational Science and Engineering, , pp. 3-21, Springer, 2004.

[18] P. Imkeller and A. H. Monahan, "Conceptual stochastic climate models," Stochastics and Dynamics, vol. 2, no. 3, pp. 311-326, 2002.

[19] B. Guo, Y. Lv, and X. Yang, "Dynamics of stochastic Zakharov equations," Journal of Mathematical Physics, vol. 50, no. 5, article 052703, 2009.

[20] Y. Guo, B. Guo, and D. Li, "Asymptotic behavior of stochastic dissipative quantum Zakharov equations," Stochastics and Dynamics, vol. 13, no. 2, article 1250016, 2013.

[21] J. L. Lions and E. Macenes, Proble' mes aux Limites Non Homoge' nes et Applications, Dunod, 1969.

[22] G. Da Prato and J. Zabczyk, Stochastic Equations in Infinite Dimensions, Cambridge University Press, 1992.

[23] R. Temam, Infinite-Dimensional Dynamical Systems in Mechanics and Physics, Springer-Verlag, 2nd edition, 1997. 